\title{
ILC2-modulated T cell-to-MDSC balance is associated with bladder cancer recurrence
}

\author{
Mathieu F. Chevalier, ${ }^{1}$ Sara Trabanelli, ${ }^{2}$ Julien Racle, ${ }^{3,4}$ Bérengère Salomé, ${ }^{2}$ Valérie Cesson, ${ }^{1}$ Dalila Charbi, ${ }^{1}$ Perrine Bohner, \\ Sonia Domingos-Pereira, ${ }^{1}$ Florence Dartiguenave, ${ }^{1}$ Anne-Sophie Fritschi, ${ }^{1}$ Daniel E. Speiser, ${ }^{5}$ Cyrill A. Rentsch, ${ }^{6}$ \\ David Gfeller, ${ }^{3,4}$ Patrice Jichlinski,, Denise Nardelli-Haefliger, ${ }^{1}$ Camilla Jandus, ${ }^{2}$ and Laurent Derré \\ 'Urology Research Unit, University Hospital of Lausanne (CHUV), Lausanne, Switzerland. ${ }^{2}$ Translational Tumor Immunology, Ludwig Center for Cancer Research at the University of Lausanne, \\ Department of Fundamental Oncology, Epalinges, Switzerland. ${ }^{3}$ Computational Cancer Biology, Ludwig Center for Cancer Research at the University of Lausanne, Epalinges, Switzerland. \\ ${ }^{4}$ Swiss Institute of Bioinformatics (SIB), Lausanne, Switzerland. 5epartment of Oncology and Ludwig Center for Cancer Research, University of Lausanne, Epalinges, Switzerland. \\ ${ }^{6}$ Department of Urology, University Hospital of Basel, Basel, Switzerland.
}

\begin{abstract}
Non-muscle-invasive bladder cancer (NMIBC) is a highly recurrent tumor despite intravesical immunotherapy instillation with the bacillus Calmette-Guérin (BCG) vaccine. In a prospective longitudinal study, we took advantage of BCC instillations, which increase local immune infiltration, to characterize immune cell populations in the urine of patients with NMIBC as a surrogate for the bladder tumor microenvironment. We observed an infiltration of neutrophils, T cells, monocytic myeloidderived suppressor cells (M-MDSCs), and group 2 innate lymphoid cells (ILC2). Notably, patients with a T cell-to-MDSC ratio of less than 1 showed dramatically lower recurrence-free survival than did patients with a ratio of greater than 1 . Analysis of early and later time points indicated that this patient dichotomy existed prior to BCC treatment. ILC2 frequency was associated with detectable IL-13 in the urine and correlated with the level of recruited M-MDSCs, which highly expressed IL-13 receptor $\alpha 1$. In vitro, ILC2 were increased and potently expressed IL-13 in the presence of BCC or tumor cells. IL-13 induced the preferential recruitment and suppressive function of monocytes. Thus, the T cell-to-MDSC balance, associated with a skewing toward type 2 immunity, may predict bladder tumor recurrence and influence the mortality of patients with muscle-invasive cancer. Moreover, these results underline the ILC2/IL-13 axis as a targetable pathway to curtail the M-MDSC compartment and improve bladder cancer treatment.
\end{abstract}

\section{Introduction}

Interactions between cancer and immunity have been intensively studied during the past 2 decades $(1,2)$. However, mechanisms hindering efficient constitutive and/or treatment-induced immune responses to human tumors have yet to be closely scrutinized. With rising incidence rates, bladder cancer (BCa) represents the fourth most commonly diagnosed cancer in men in Europe and the United States (3). Notably, BCa is the most expensive malignancy to treat per patient (4), mostly on account of dramatically high recurrence rates requiring lifelong follow-up with multiple surgical procedures. Most patients (>70\%) present with a non-muscle-invasive $\mathrm{BCa}$ (NMIBC) at diagnosis, i.e., cancer that is confined to the mucosa (stage Ta, carcinoma in situ [CIS]) or submucosa (stage T1) (5). A major aim in the management of urothelial carcinoma is the prevention of the recurrence and progression of disease. For patients with an intermediate to high risk of recurrence, the standard treatment consists of intravesical instillations with the bacillus Calmette-Guérin (BCG) to prevent

Authorship note: M.F. Chevalier and S. Trabanelli contributed equally to this work. L. Derre and $C$. Jandus equally contributed to this work as co-senior authors.

Conflict of interest: The authors have declared that no conflict of interest exists.

Submitted: July 21, 2016; Accepted: May 4, 2017

Reference information: / Clin Invest. 2017;127(8):2916-2929.

https://doi.org/10.1172/JCI89717. or delay tumor recurrence and progression (5). However, 20\% to $30 \%$ of patients experience early recurrence, despite BCG treatment, and eventually undergo surgical removal of the bladder $(5,6)$. Several decades after this empirical immunotherapy was approved by the US FDA (7), the mode of action of intravesical BCG therapy remains unclear (8). Yet, it was shown that the strong inflammation induced locally encompasses (a) secretion of various chemokines and cytokines (9) and (b) infiltration of innate and adaptive immune cells, mostly neutrophils, T cells, and monocytic cells (8). This highlights the crucial need to better understand the regulation of immune responses that occur during the treatment of patients with NMIBC in order to identify new targetable pathways to improve BCa therapy.

In this prospective study, we performed a comprehensive analysis of immune cells in the urine of patients during BCG therapy, which increases the immune cell content locally. We found that the ratio of $\mathrm{T}$ cells to monocytic myeloid-derived suppressor cells (M-MDSCs), known to dampen antitumor immunity $(10,11)$, is strongly associated with recurrence-free survival outcomes. Of note, BCG treatment does not seem to alter this ratio. We also identified, for the first time to our knowledge in a human cancer, a putative role for group 2 innate lymphoid cells (ILC2), which were recently described as the innate counterpart of Th2 cells $(12,13)$. ILC2 levels correlated with M-MDSC levels in urine upon treatment with BCG. In vitro, bladder tumor cells and BCG were able 


\section{Table 1. Characteristics of NMIBC patients}

\begin{tabular}{|c|c|c|c|c|}
\hline Characteristics & All patients & Low $T$ cell/MDSC ratio & High T cell/MDSC ratio & $P$ value \\
\hline No. of patients & 28 & 14 & 14 & \\
\hline Age, yr, median (IQR) & $68(62-74)$ & $68.5(63-80)$ & $67(60-73)$ & $\operatorname{NS}(P=0.394)^{A}$ \\
\hline Sex, $n$ & & & & NS $(P=1.0)^{\mathrm{B}}$ \\
\hline Male & 24 & 12 & 12 & \\
\hline Female & 4 & 2 & 2 & \\
\hline Previous bladder tumor history, $n$ (\%) & & & & NS $(P=0.641)^{\mathrm{c}}$ \\
\hline No previous TURB & $18(64.3)$ & $8(57.1)$ & $10(71.4)$ & \\
\hline 1 previous TURB & $6(21.4)$ & $4(28.6)$ & $2(14.3)$ & \\
\hline$\geq 2$ previous TURB & $4(14.3)$ & $2(14.3)$ & $2(14.3)$ & \\
\hline No. of second TURB, $n(\%)$ & $23(82.1)$ & $11(78.6)$ & $11(78.6)$ & $\operatorname{NS}(P=1.0)^{\mathrm{B}}$ \\
\hline No. of positive second TURB, $n$ (\%) & $12(42.9)$ & $7(63.6)$ & $4(33.3)$ & NS $(P=0.395)^{\mathrm{B}}$ \\
\hline Tumor status, $n(\%)$ & & & & $\operatorname{NS}(P=0.705)^{c}$ \\
\hline pTa & $4(14.3)$ & $2(14.3)$ & $3(21.4)$ & \\
\hline pT1 & $20(71.4)$ & $11(78.6)$ & $9(64.3)$ & \\
\hline pTIS & $3(10.7)$ & $1(7.1)$ & $1(7.1)$ & \\
\hline pT2 & $1^{0}(3.6)$ & 0 & $1(7.1)$ & \\
\hline Any CIS-positive & $17(60.7)$ & $8(57.1)$ & $9(64.3)$ & $\operatorname{NS}(P=1)^{B}$ \\
\hline \multicolumn{5}{|l|}{ Grade, $n(\%)$} \\
\hline G2 low grade & $3(10.7)$ & $1(7.1)$ & $2(14.3)$ & NS $(P=0.487)^{\mathrm{C}}$ \\
\hline G2 high grade & $11(39.3)$ & $7(50)$ & $4(28.6)$ & \\
\hline G3 & $14(50)$ & $6(42.9)$ & $8(57.1)$ & \\
\hline No. of tumors & & & & NS $(P=0.606)^{c}$ \\
\hline 1 & $12(42.9)$ & $5(35.7)$ & $7(50)$ & \\
\hline 2 & $10(35.7)$ & $5(35.7)$ & $5(35.7)$ & \\
\hline$\geq 3$ & $6(21.4)$ & $4(28.6)$ & $2(14.3)$ & \\
\hline
\end{tabular}

to shift ILCs toward the ILC2 phenotype. Moreover, in response to BCG, ILC2 produced the type 2 cytokine IL-13, which mediated the recruitment and immunosuppressive function of monocytic cells. These results were corroborated by gene expression and patient survival analysis derived from The Cancer Genome Atlas (TCGA) data. Altogether, our findings identify a previously unknown mechanism of BCG failure and suggest the existence of a new innate ILC2/MDSC axis that fosters an immunosuppressive tumor microenvironment.

\section{Results}

Characterization of immune cells present in the urine during BCG therapy. An easy means for monitoring immune events in the bladder is to examine immune cells present in urine collected shortly after immunotherapeutic interventions. Thus, we performed a comprehensive phenotypic analysis of immune cells in urine samples from 28 patients with NMIBC during a standard 6-week intravesical BCG therapy. The patients' characteristics are described in Table 1. Urine samples were obtained before and 4 hours after each instillation. As expected, most of the recruited cells were neutrophils (Figure 1, A and B), but we also detected T cells (Figure 1, A and C) (with variable CD4/CD8 ratios between samples and patients; data not shown), as well as monocytic cells (Figure 1D). Interestingly, a substantial number of urine CD14 ${ }^{+}$cells showed an M-MDSC phenotype (i.e., $\left.\mathrm{CD} 15^{-} \mathrm{CD} 14^{+} \mathrm{CD} 11 \mathrm{~b}^{+} \mathrm{CD} 33^{+} \mathrm{HLA}^{-D R^{\mathrm{lo}}}\right)(10,11)$. These cells were sorted (Supplemental Figure 1; supplemental material available online with this article; https:// doi.org/10.1172/JCI89717DS1), and mRNA was extracted for quantitative PCR (qPCR) analyses. As shown in Figure 1E, CD14 CD $33^{+}{ }^{H L A}-\mathrm{DR}^{\text {lo }}$ urine-sorted cells were found to express higher levels of CCAAT/enhancer-binding protein $\beta(\mathrm{C} / \mathrm{EBP} \beta)$, an MDSCassociated transcription factor governing their suppressive function (14), compared with their CD33 ${ }^{+}$ HLA-DR ${ }^{\text {hi }}$ counterparts. Accordingly, Cebpb mRNA levels correlated with those of both arginase 1 (ARG1) and inducible NOS (iNOS), 2 enzymes that are classically involved in MDSC-suppressive activity (Figure 1F), thus confirming the M-MDSC phenotype.

While the mean frequency of each cell subset did not significantly change during the 6-week follow-up (Figure 1, B, C, and G), the absolute numbers of these cells increased in parallel with the numbers of cells in the urine (Figure $1 \mathrm{H}$ ). Of note, the latter were higher in post- versus pre-BCG samples, confirming that BCG induces a strong local inflammation involving a variety of immune cells (Figure $1 \mathrm{H}$ ).

To address the possible involvement of bladder tumors or BCG in M-MDSC induction, peripheral blood mononuclear cells (PBMCs) from healthy donors (HDs) were cocultured with Bu68.8 (non-muscle-invasive) or T24 (muscle-invasive) BCa cell lines, or infected with different MOI of BCG. Both cell lines, as well as BCG in a dose-dependent manner, induced a downregulation of HLA-antigen D-related (HLA-DR) on monocytes, leading to an increase in the frequency of cells with an M-MDSC phenotype (Supplemental Figure 2, A and B). Of note, heat-killed BCG had a similar effect, albeit to a lesser extent, suggesting that infection may not be required (Supplemental Figure 2C). Moreover, to investigate whether the skewing toward M-MDSCs is specific to BCG, we tested the only other FDA-approved live attenuated bacterial vaccine, i.e., the Salmonella typhi strain Ty21a (which we recently showed to be more efficient than BCG for bladder tumor treatment in a mouse model [ref. 15]). As shown in Supplemental Figure 2C, Ty21a bacteria had a limited effect on the induction of M-MDSCs.

In order to assess the function of induced M-MDSCs, CD14 ${ }^{+}$ cells were sorted from the coculture with tumor cell lines and used in a classical $\mathrm{T}$ cell suppression assay. In contrast to untreated monocytes, $\mathrm{CD} 14^{+}$cells previously cocultured with $\mathrm{T} 24$ or Bu68.8 cell lines exerted a strong suppressive function toward CD8 and CD4 T cell proliferation (Supplemental Figure 2D). 
A

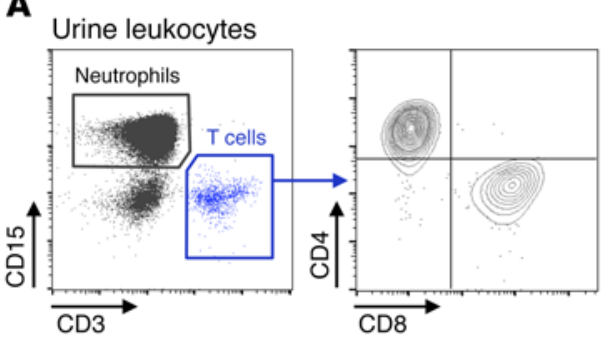

B

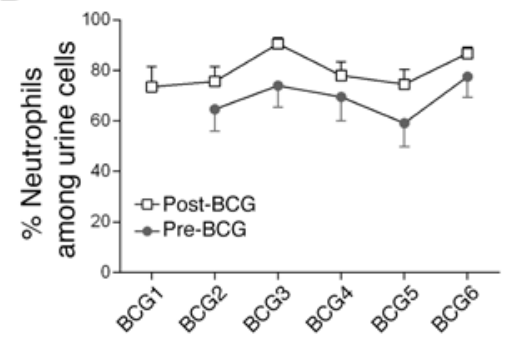

C

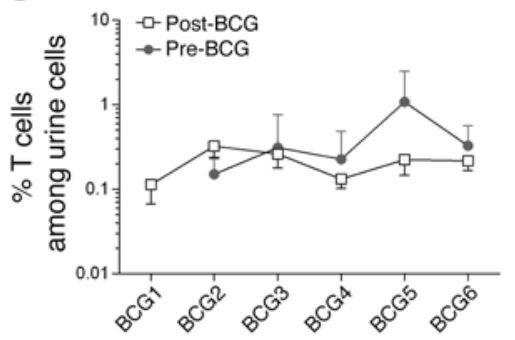

D

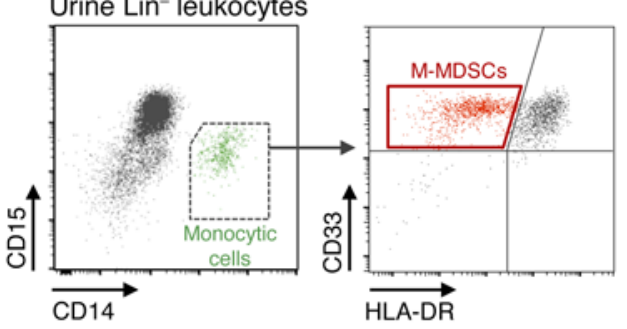

E

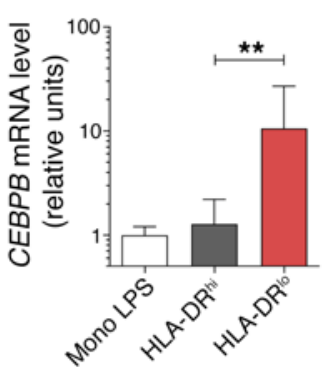

$\mathbf{F}$

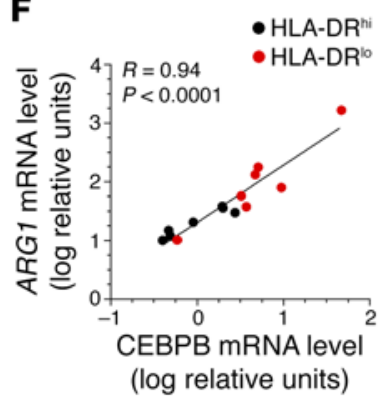

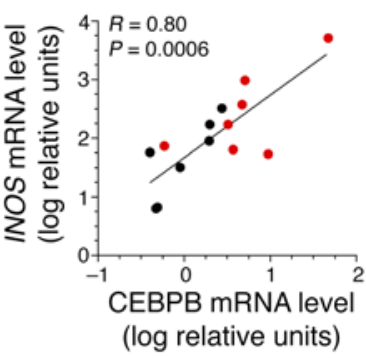

G

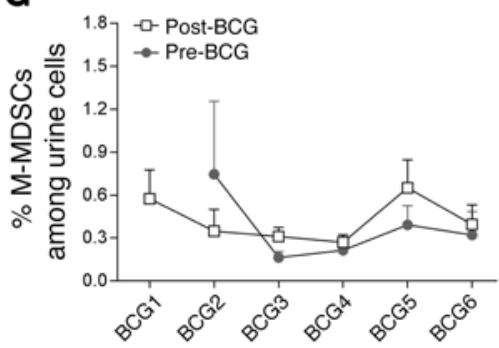

H

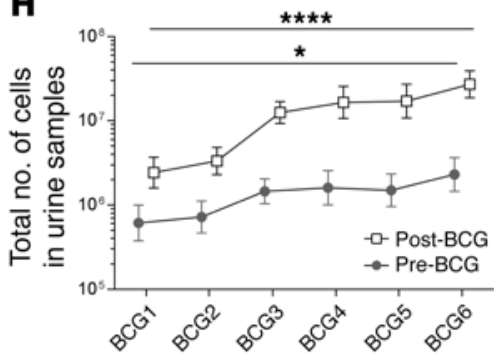

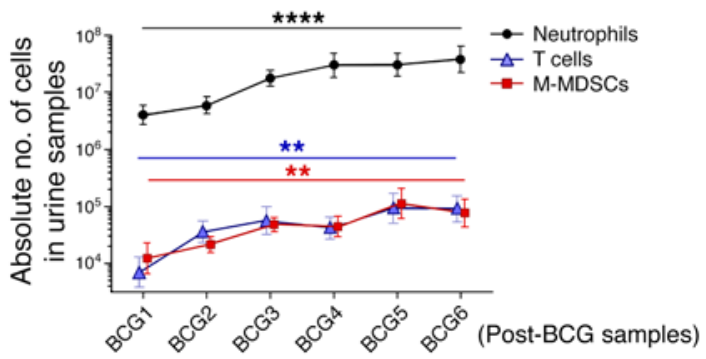

Figure 1. Identification of immune cells infiltrating the urine during BCG therapy. Flow cytometric analysis of urine-infiltrating cells in urine samples obtained from 28 patients with NMIBC during the 6-week intravesical BCC therapy. Urine samples were obtained before and 4 hours after each BCG instillation. Pre-BCC1 data are not shown due to low urine cell content, which is typical at that time point and does not allow for measurement of immune cell subsets in most patients. (A) Samples were gated on live leukocytes and neutrophils (CD15+), and CD3 $3^{+} T$ cells (comprising both CD4 ${ }^{+}$and CD8 $8^{+} T$ cells) were assessed. (B and $\mathbf{C}$ ) The frequencies (mean \pm SEM) of both cell subsets in urine are depicted during the follow-up period. (D) When gating on lineage-

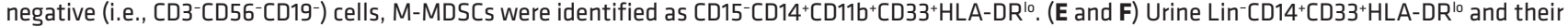
HLA-DR ${ }^{\text {hi }}$ counterpart cells were sorted from 7 urine samples, and mRNA levels of CEBPB, $A R G 1$, and INOS were measured relative to the levels detected in circulating LPS-activated monocytes from HDs $(n=3)$. Levels (mean \pm SEM) of CEBPB are shown for the indicated cell subsets $(E)$ and correlated with ARG1 and INOS mRNA levels (F). A 2-tailed, paired Student's $t$ test was performed to compare both cell populations, and Spearman's rank correlation coefficients $(R)$ and the corresponding $P$ values are indicated on each panel in $\mathbf{F}$. Lines indicate linear regression. (C) Frequencies (mean \pm SEM) of urine M-MDSCs during the follow-up period. (H) Graphs show the mean ( \pm SEM) of total cell numbers in pre- and post-BCC urine samples and absolute numbers of the indicated cells subsets in post-BCG samples. ${ }^{*} P<0.05$, ${ }^{*} P<0.01$, and ${ }^{* * *} P<0.0001$, by 1 -way ANOVA, followed by a post test for linear trend to assess longitudinal changes.

The local T cell/MDSC balance is strongly associated with bladder tumor recurrence. To evaluate whether and what immune profiles may be involved in BCG treatment failure, we prospectively followed the time to tumor recurrence or progression, if any, for each of the 28 patients. The median follow-up after the start of BCG treatment was 33.6 months, and the 2-year recurrence-free survival rate was $56.0 \%$ (95\% CI, 34.2\%-73.1\%) (Supplemental Figure $3 \mathrm{~A})$. Patients show similar recurrence-free survival as previously described in larger cohorts of patients treated with the same BCG strain (TICE) $(6,16)$. When we segregated patients into groups on the basis of urine frequency of neutrophils (Figure 2A), T cells (Figure 2B), and M-MDSCs (Figure 2C) (i.e., mean percentage in postBCG urine samples greater than versus less than the median), we found no significant difference in recurrence-free survival rates, although we observed trends for T cells and M-MDSCs. As the balance between effector and regulatory cells may be more relevant $(17,18)$, we then assessed for each patient the mean T cell/MDSC ratio in post-BCG urine samples. We segregated the patients into 2 groups: (a) patients with higher frequencies of M-MDSCs than $\mathrm{T}$ cells (i.e., $\log$ ratio $<0, n=14$ ) and (b) patients with higher frequencies of T cells than M-MDSCs (i.e., log ratio $>0, n=14$ ) (Figure 2D). Of note, the ratio measured at the earliest time point available (i.e., pre-BCG 1 or 2) correlated with ratios obtained at later time points (the mean of all post-BCG samples) from the same patients (Figure $2 \mathrm{E}$ ), suggesting that the patient dichotomy based on the postBCG T cell/MDSC ratios already existed before BCG treatment initiation and arguing in favor of a prognostic role of the T cell/ MDSC ratio. Notably, we found that the 2 groups strongly differed 

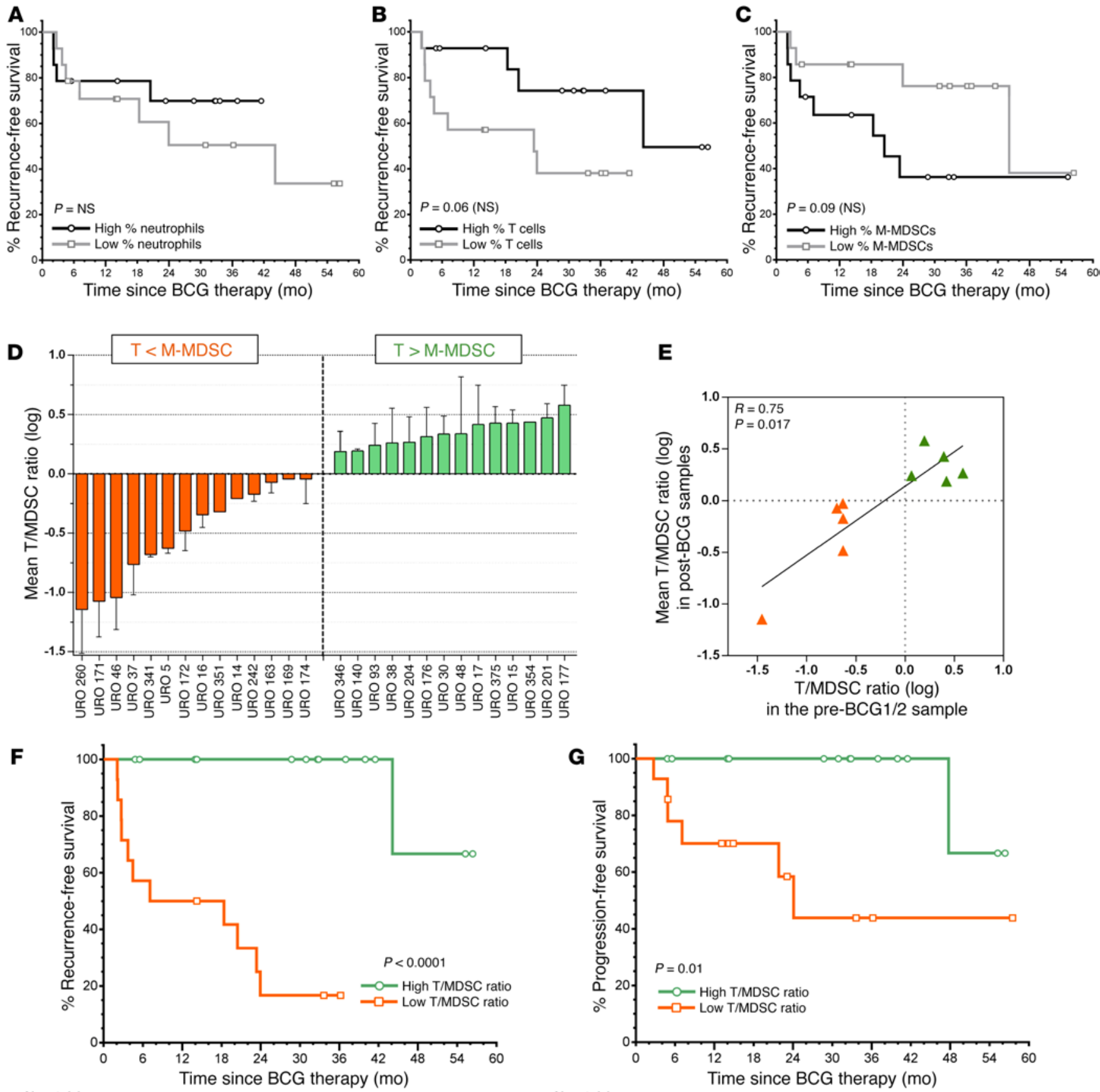

No. at risk:

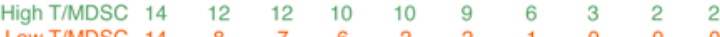

No. at risk:

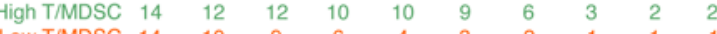

Figure 2. Recurrence-free and progression-free survival in patient groups following BCG therapy for NMIBC. Recurrence-free survival was assessed using the Kaplan-Meier approach in a cohort of 28 patients receiving BCG therapy (with the first instillation at month 0 and the last at month 1.5). Censored patients are represented by symbols. Patients were segregated into 2 groups ( $n=14$ patients in each group) on the basis of urine levels of neutrophils (A), T cells (B), or M-MDSCs (C), i.e., the mean percentage of $\mathrm{CD}^{2} 5^{+}$, the mean percentage of $\mathrm{CD}^{+}$, or the mean percentage of Lin-CD14 CD11b+CD33+HLA-DR ${ }^{10}$ cells above the median (High) versus below the median (Low) in post-BCG samples (2-6 time points per patient). (D) The mean T cell/MDSC ratio in post-BCG urine samples identified 2 groups: patients with higher frequencies of M-MDSCs than of T cells (i.e., log ratio $<0$ ) and conversely (i.e., log ratio $>0$ ). (E) The T cell/MDSC ratio was measurable at early time points before BCG (1 or 2 ) for 10 patients and correlated with the mean $T$ cell/MDSC ratios in post-BCG samples from corresponding patients. The line indicates linear regression, and Spearman's rank correlation coefficients $(R)$ and the corresponding $P$ value are indicated. Recurrence-free survival $(\mathbf{F})$ and progression-free survival $(\mathbf{G})$ according to the T cell/MDSC ratio. $P$ values from log-rank tests are indicated on each panel. T, T cell. 
A

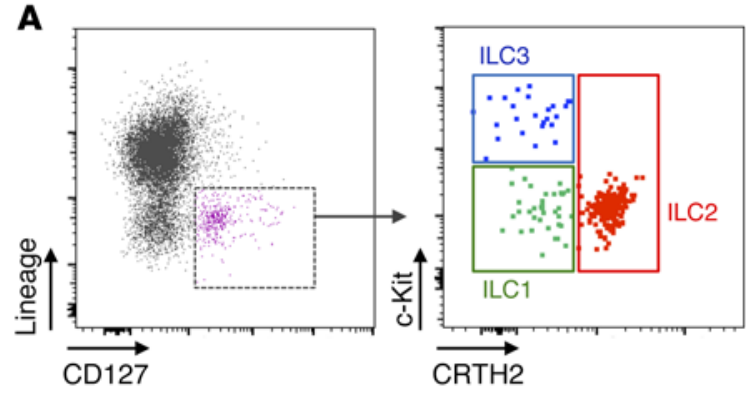

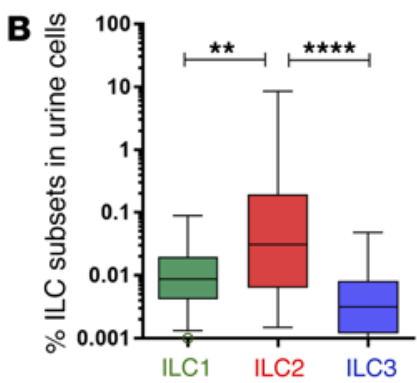
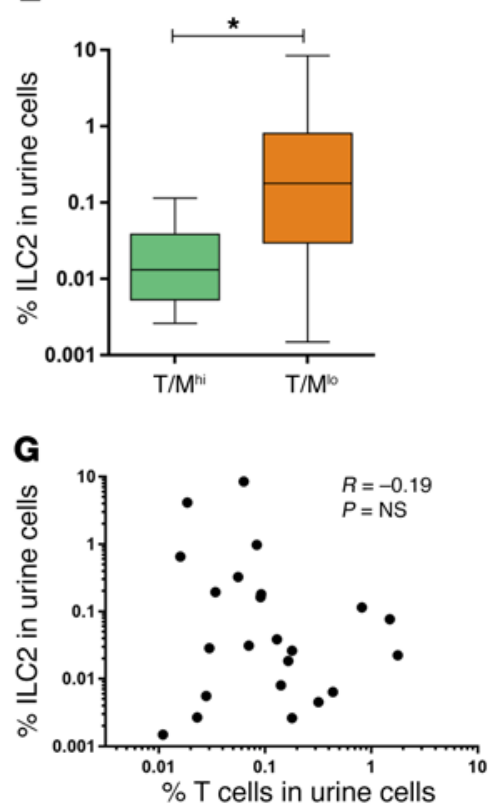

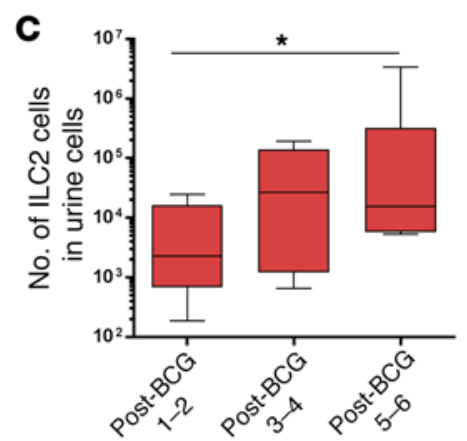

$\mathbf{F}$
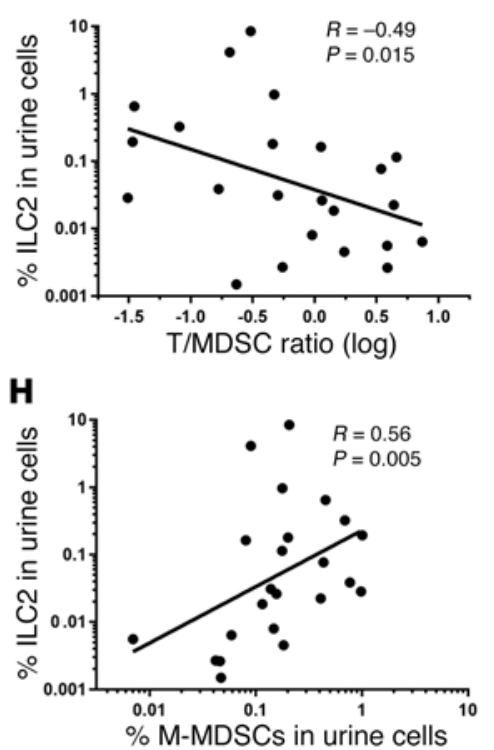

Figure 3. Skewing toward ILC2 is associated with low T cell/MDSC ratios during BCG therapy for NMIBC. (A) Representative example of ILC labeling. Cells

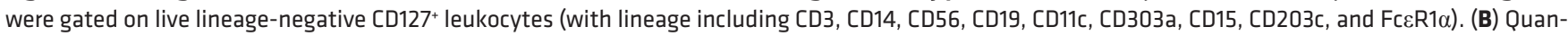
tification of ILC1 (Lin-CD127+CRTH2-c-Kit'), ILC2 (Lin-CD127+CRTH2 ${ }^{+}$), and ILC3 (Lin-CD127+CRTH2- c-Kit ${ }^{+}$) among urine cells ( $n=23$ samples). (C) Absolute number of ILC2 in post-BCG samples. (D) Cocultures of PBMCs from HDs $(n=6)$ with the NMIBC cell line Bu68.8, BCC (MOI = 1$)$, or medium alone for 4 days. Repartition of ILC1, ILC2 and ILC3 subsets as well as frequencies of total ILC are shown for each indicated conditions. (E) ILC2 frequencies in post-BCC urine samples from patients with high ( $\mathrm{T} / \mathrm{M}^{\mathrm{hi}}, n=10$ ) or low (T/M $\left.\mathrm{M}^{\mathrm{lo}}, n=13\right) \mathrm{T}$ cell/MDSC ratios, as identified in Figure 2, were compared (2-tailed Student's $t$ test). ILC2 frequencies were correlated with the T cell/MDSC ratios (F) as well as T cell (G) and M-MDSC (H) frequencies in corresponding urine samples. Lines indicate linear regression. Spearman's rank correlation coefficients $(R)$ and the corresponding $P$ values are indicated in $\mathbf{F}-\mathbf{H} .{ }^{*} P<0.05$, ${ }^{* *} P<0.01$, and ${ }^{* * * *} P<0.0001$, by 1-way ANOVA followed by Tukey's test (B), a post test for linear trend (C), or Dunnett's test (D) for comparison of subsets, longitudinal changes, or conditions versus medium, respectively.

in terms of recurrence rates $(\log$ rank: $P<0.0001)$, as the median recurrence-free survival time following the start of BCG treatment was only 12.7 months in patients with a low T cell/MDSC ratio (T/ $\mathrm{M}^{\mathrm{lo}}$ ), while it was not reached in patients with a high T cell/MDSC ratio $\left(\mathrm{T} / \mathrm{M}^{\mathrm{hi}}\right.$ ) (median follow-up of 32.7 months in this group) (Figure $2 \mathrm{~F}$ ). We also assessed the time to progression (Supplemental Figure 3B). Five patients from the $\mathrm{T} / \mathrm{M}^{\mathrm{lo}}$ group showed tumor progression versus one patient in the other group (Figure $2 \mathrm{G}$ ).

Type 2 immunity skewing in patients with low $T$ cell/MDSC ratios. To better define the immunological profiles of these 2 patient groups with very distinct recurrence-free survival rates, we analyzed Th cells (Th1 versus Th2 polarization), as well as their innate counterparts (i.e., including the ILCs ILC1, ILC2, and ILC3). Freshly isolated urine T cells were expanded in vitro, and
CD4 T cells were analyzed for expression of Th1 and Th2 markers as well as for cytokine secretion profiles. While the proportions of $\mathrm{CRTH} 2^{+} \mathrm{CD} 4 \mathrm{~T}$ cells were low in urine samples, they were found at higher levels in patients from the $\mathrm{T} / \mathrm{M}^{\mathrm{lo}}$ group (Supplemental Figure 4A). As expected, we detected high levels of the Th1 cytokines IFN- $\gamma$, IL-2, and TNF- $\alpha$ and low levels of the Th2 cytokines IL-4 and IL-5 in the supernatants of urine $\mathrm{CD}^{+} \mathrm{T}$ cells following 24-hour anti-CD3/CD28 stimulation (data not shown). Notably, patients in the $\mathrm{T} / \mathrm{M}^{\mathrm{lo}}$ group showed a higher $\mathrm{Th} 2$ to Th1 cytokine score (Supplemental Figure 4B). Moreover, since the Th1/Th2 balance may be regulated by tryptophan (Trp) metabolism $(19,20)$, we assessed local indoleamine 2,3 dioxygenase (IDO) activity by measuring the kynurenine/Trp ratio in pre- and post-BCG urine samples (at the fifth instillation). Accordingly, we found that the 

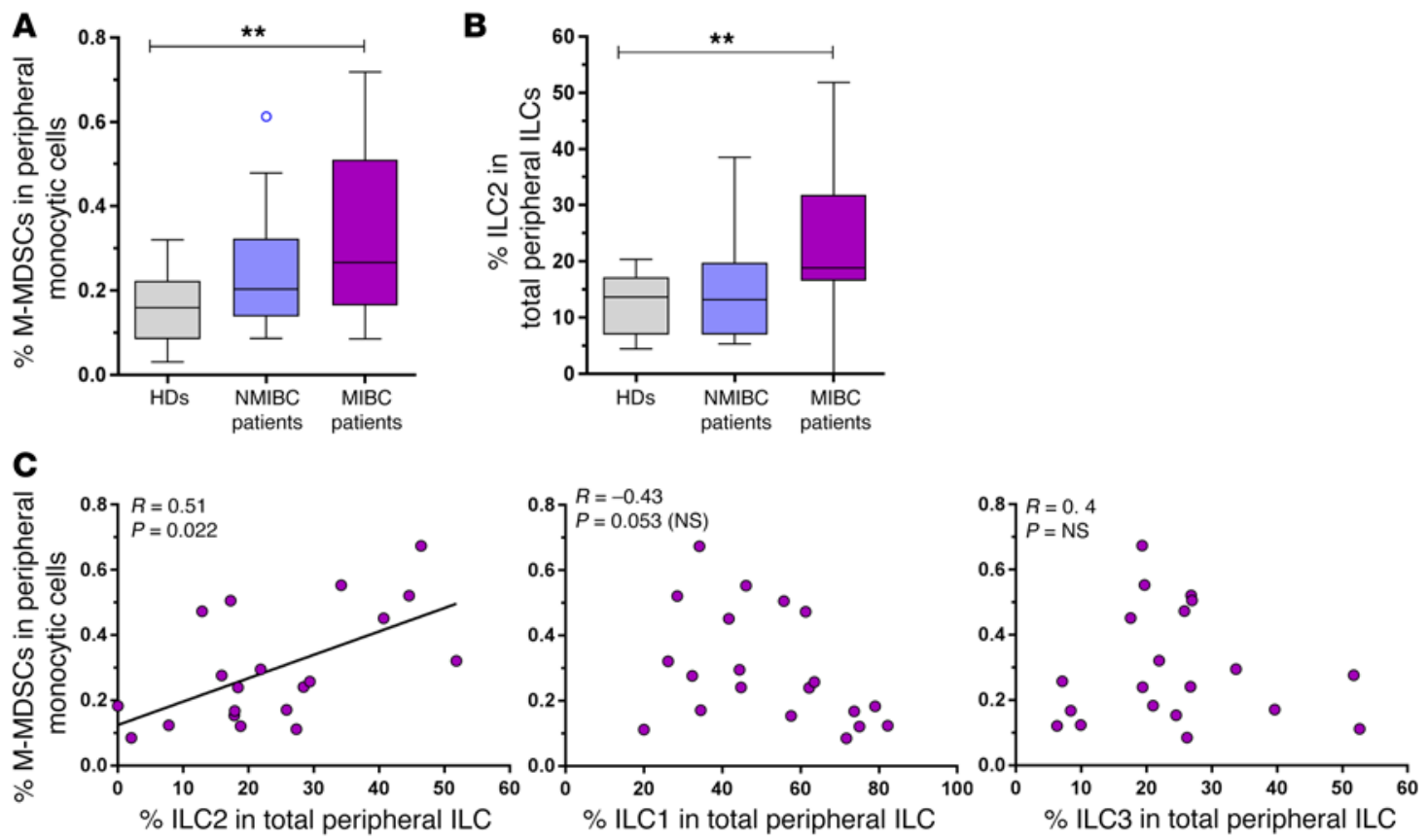

Figure 4. Peripheral M-MDSCs and ILC2 in blood from NMIBC and MIBC patients. (A and B) Ex vivo frequencies of (A) M-MDSCs (Lin-CD14 ${ }^{+} C D 33^{+} C D 11 b^{+}$ HLA-DR ${ }^{10}$ cells) and (B) ILC2 (Lin-CD127+CRTH2+) measured in PBMCs from HDs $(n=14)$, patients with NMIBC $(n=18)$, and patients with MIBC at the time of cystectomy ( $n=22$ and $n=21$ for M-MDSCs and ILC2, respectively). ${ }^{* *} P<0.01$, by 1-way ANOVA followed by Dunnett's test. (C) Peripheral M-MDSC levels were correlated with those of the indicated ILC subsets $(n=20)$. Lines indicate linear regression. Spearman's rank correlation coefficients $(R)$ and the corresponding $P$ values are indicated.

level of IDO activity was higher in samples from patients in the $\mathrm{T} / \mathrm{M}^{\mathrm{lo}}$ group (Supplemental Figure $4 \mathrm{C}$ ).

We next analyzed the presence and repartition of ILCs in urine samples from patients during their BCG therapy. While the levels of type 1 and type 3 ILCs were low, we found measurable levels of ILC2 ( $\mathrm{Lin}^{-} \mathrm{CD} 127^{+} \mathrm{CRTH} 2^{+}$cells) in the urine (Figure $3, \mathrm{~A}-\mathrm{C}$ ). In consonance with this observation, in vitro experiments using PBMCs from HDs (Figure 3D) showed that BCa cells and BCG were able to significantly augment ILC2 proportions among total ILCs, at the expense of ILC3 and ILC1, respectively. Moreover, only BCG tended to induce an increase in total ILC frequencies (Figure 3D). Of note, in contrast to Ty21a, heat-killed BCG also significantly increased the percentage of ILC2, albeit to a lesser extent compared with the percentage of ILC2 with live BCG (Supplemental Figure 5).

We also sought evidence to explain how ILC2 could infiltrate the bladder in this context. It was recently reported that ILC2 expressed the chemokine receptor CCR4 and did so at higher proportions than was observed with ILC1 or ILC3 (21). We confirmed that CCR4 was expressed on peripheral ILC2 from patients with NMIBC, and at a significantly higher frequency than was observed on ILC1 or ILC3 (Supplemental Figure 6A). Notably, ligands for CCR4 (macrophage inflammatory protein $1 \alpha$ [MIP-1 $\alpha$ ], monocyte chemoattractant protein 1 [MCP-1], regulated on activation, normal T cell expressed and secreted [RANTES, also known as CCL5], and CCL22 [also known as MDC]) have been reported to be induced locally during BCG therapy $(9,22-25)$. Of note, we were able to measure CCR4 expression in ILCs from 3 urine samples during BCG therapy (Supplemental Figure 6B) and found that most ILC2 but also, in contrast to the periphery, most ILC1 and
ILC3 expressed CCR4 in the urine. Altogether, these data suggest that CCR4 may be involved in the recruitment of ILCs in the bladder during BCG therapy, with a preferential enrichment in ILC2, since ILC2, compared with ILC1 and ILC3, express a higher proportion of CCR 4 in the periphery. Besides, bladder tumor cells are able to produce low levels of prostaglandin $\mathrm{D}_{2}$ (PGD2) (Supplemental Figure 6C), a chemoattractant for ILC2 (26), which could also contribute to the recruitment of ILC2 in the bladder, as shown in the inflamed lung (27).

Notably, patients in the $\mathrm{T} / \mathrm{M}^{\mathrm{lo}}$ group showed higher levels of ILC2 in the urine (Figure 3E). The negative correlation between the T cell/MDSC ratio and ILC2 levels (Figure 3F) is probably explained by a putative link between ILC2 and M-MDSCs, as ILC2 levels positively correlated with M-MDSCs but not with $\mathrm{T}$ cells (Figure 3, G and H). Altogether, these results suggest that local type 2 immunity skewing is linked to the immune profile associated with BCG treatment failure.

We next evaluated ILC2 and M-MDSCs in blood from the same patients (i.e., BCG-treated NMIBCs) but also from patients with more advanced (i.e., muscle-invasive $\mathrm{BCa}[\mathrm{MIBC}]$ ) at the time of cystectomy (Supplemental Table 1). As compared with HDs, patients with NMIBC had normal levels of circulating M-MDSCs and ILC2 (Figure 4, A and B), with no difference between the $\mathrm{T} / \mathrm{M}^{\mathrm{lo}}$ and $\mathrm{T} / \mathrm{M}^{\mathrm{hi}}$ groups (data not shown). In contrast, patients with MIBC showed increased peripheral blood levels of both cell subsets (Figure 4, A and B). Of note, the proportion of ILC2, but not ILC1 or ILC3, was positively correlated with M-MDSC levels in the blood of MIBC patients (Figure 4C), which substantiates the observations in urine. 
Potential involvement of an ILC2/IL-13/MDSC axis in BCG failure. We tested the presence of ILC2-associated cytokines in urine samples. While IL-4 and IL-5 were undetectable (data not shown), IL-13 was detected in 6 of 13 post-BCG samples corresponding to patients with higher frequencies of ILC2 in the urine as compared with those with IL-13 levels below the detection limit (Figure 5A). We then performed an in vitro evaluation of the propensity of ILC2 to produce IL-13 in the context of BCG stimulation. PBMCs from both HDs and patients with MIBC, who showed elevated ILC2 levels (Figure 4B), were stimulated with BCG for 48 hours, and we then measured IL-13 production in ILC2 (Figure 5B). Interestingly, ILC2 were strongly able to express IL-13 in response to BCG, with higher proportions found in ILC2 from MIBC patients compared with ILC2 from HDs (Figure 5C). Besides, in contrast to nonmalignant urothelium (HCV-29 cell line) and to the Bu68.8 cell line, the MIBC cell line (TCC-Sup) significantly upregulated IL-13 expression in ILC2 from patients with MIBC (Figure 5D).

Hence, we next investigated the potential contribution of an ILC2/IL-13-mediated mechanism in M-MDSC recruitment and/ or induction. While IL-13 is known to alternatively activate macrophages (M2) (28), the role of IL-13 in MDSC biology in humans remains elusive. To consider a potential effect of IL-13 on human M-MDSCs, we first examined whether these cells express the IL-13 receptor $\alpha 1$ (IL-13R $\alpha 1$ ) (Figure 5E), as was recently suggested in a mouse study (29). As shown in Figure 5F, monocytic cells from both HDs and patients expressed IL-13R $\alpha 1$. We observed a higher receptor density (a) on M-MDSCs compared with monocytes and (b) in urine compared with circulating M-MDSCs. We then investigated the role of IL-13 on monocytic cells. First, we found in a Transwell experiment using PBMCs from HDs that IL-13 was able to preferentially attract monocytes in contrast to lymphocytes (Figure 5G), as suggested in a previous study (30). We next functionally assessed whether IL-13 may confer suppressive activity to monocytic cells. Therefore, blood monocytes isolated from HDs were cultured in the presence of granulocyte macrophageCSF (GM-CSF) (as a survival factor) for 4 days, with or without recombinant IL-13. Cells were subsequently used in autologous $\mathrm{T}$ cell proliferation assays. Analysis of CFSE dilution in cocultured $\mathrm{T}$ cells stimulated by anti-CD3/CD28 showed that IL-13-treated $\mathrm{CD}_{14}{ }^{+}$cells were able to significantly inhibit the proliferation of both $\mathrm{CD}^{+}$and $\mathrm{CD} 8^{+} \mathrm{T}$ cells, in contrast to untreated $\mathrm{CD} 14^{+}$cells (Figure 5, H-J). Phenotypic analysis of the IL-13-treated monocytic cells is shown in Supplemental Figure 7. Notably, IL-13 downregulated HLA-DR expression, thereby increasing the frequencies of HLA-DR ${ }^{\text {lo }}$ cells in sorted CD14 ${ }^{+}$cells (Supplemental Figure 7, A and B). Of note, while CD115 (CSF1-R) was upregulated following IL-13 treatment in both HLA-DR ${ }^{\text {hi }}$ and HLA-DR ${ }^{\text {lo }}$ cells, all other monocyte and macrophage surface markers tested showed lower expression, including that of the M2 marker CD163 (Supplemental Figure 7C). Therefore, it seems that the ability of IL-13 to induce suppressive function in monocytic cells is attributable to an induction of M-MDSCs rather than of M2 macrophages, although the differences between these subsets remain unclear (11). Furthermore, IL-13 transcriptionally upregulated ARG1, iNOS, and C/EBP $\beta$ (Supplemental Figure 7D), which were also found to be expressed in urine M-MDSCs from patients (Figure 1, D and E). Moreover, IL-13 treatment of $\mathrm{CD} 14^{+}$cells increased the secretion of IL-1 receptor antagonist (IL-1RA), an antiinflammatory molecule blocking IL-1 signaling, while it decreased the secretion of MCP-1 and IL-8, a neutrophil-recruiting cytokine known to be associated with successful BCG therapy in patients with BCa (31) (Supplemental Figure 7E).

To substantiate an IL-13-mediated mechanism in M-MDSC induction upon BCG treatment, we tested the effect of BCG-stimulated PBMC supernatant on monocytic cells in the absence or presence of an anti-IL-13-blocking antibody. HD PBMCs were stimulated with BCG for 3 days, and supernatants were harvested. As shown in Figure 5K, IL-13 was induced upon BCG stimulation. Filtered supernatants were then added onto sorted CD14 ${ }^{+}$ cells in the presence of either an anti-IL-13-blocking antibody or an IgG1 isotype control. After 4 days, cells were used in a $\mathrm{T}$ cell proliferation assay as above. Interestingly, we found that $\mathrm{T}$ cell proliferation, which was lower in the presence of monocytic cells conditioned by BCG-treated versus untreated supernatants, was partially recovered when IL-13 was blocked (Figure 5, L and M).

Analyses of overall survival data from TCGA. We performed a large-scale analysis of bladder urothelial cancer data from TCGA (32). In the absence of TCGA data from patients with NMIBC, this analysis confirmed our findings and extended them to patients with MIBC. These data included tumor mRNA expression and survival data from 300 patients with advanced-stage tumors (T2-T4 for $>98 \%$ of samples). We observed a counterbalancing effect of monocytic cells on T cells (appraised on the basis of CD14 and CD3E gene expression, respectively) (Figure 6A). Accordingly, short survival was associated with "low CD3, high CD14" expression and long survival with "high $C D 3$, low $C D 14$ " expression, while intermediate survival was observed in the scenarios of "low CD3, low CD14" and "high CD3, high CD14" expression (Figure 6B). Moreover, the association analysis of CD14 and IL-13 expression corroborated a link between the presence of IL-13 in the tumor and a recruitment of CD14-expressing cells (Figure 6C). Finally, a counterbalancing effect holds between T cells and the presence or absence of IL-13 in the tumor, as the shortest survival was found in patients with low $\mathrm{T}$ cell infiltration, together with detectable IL-13 (Figure 6D).

While CD3 expression accurately defines T cells, 1 single marker (CD14) is not sufficient to identify M-MDSCs, so that the influence of M-MDSCs cannot be fully deconvoluted in TCGA data. Also, one cannot exclude the possibility that IL-13 expression also captures other subtypes of immune cells that may play a role in patient survival. Nevertheless, these in silico analyses are consistent with our experimental results and suggest that the mechanisms uncovered in this study may broadly apply to both NMIBC and MIBC.

\section{Discussion}

In this prospective, longitudinal study of patients with $\mathrm{BCa}$, we observed a gradual increase in the absolute numbers of all immune cell subsets tested in urine during the course of BCG immunotherapy. In contrast to individual cell subsets, the T cell-to-MDSC ratio, likely preestablished before treatment, was associated with the clinical outcome, showing that the balance between these cell subsets may be critically involved in tumor recurrence, when tilted toward the M-MDSC side (Figure 7). For the first time to our knowledge in humans, we also identified ILC2 as new players in 

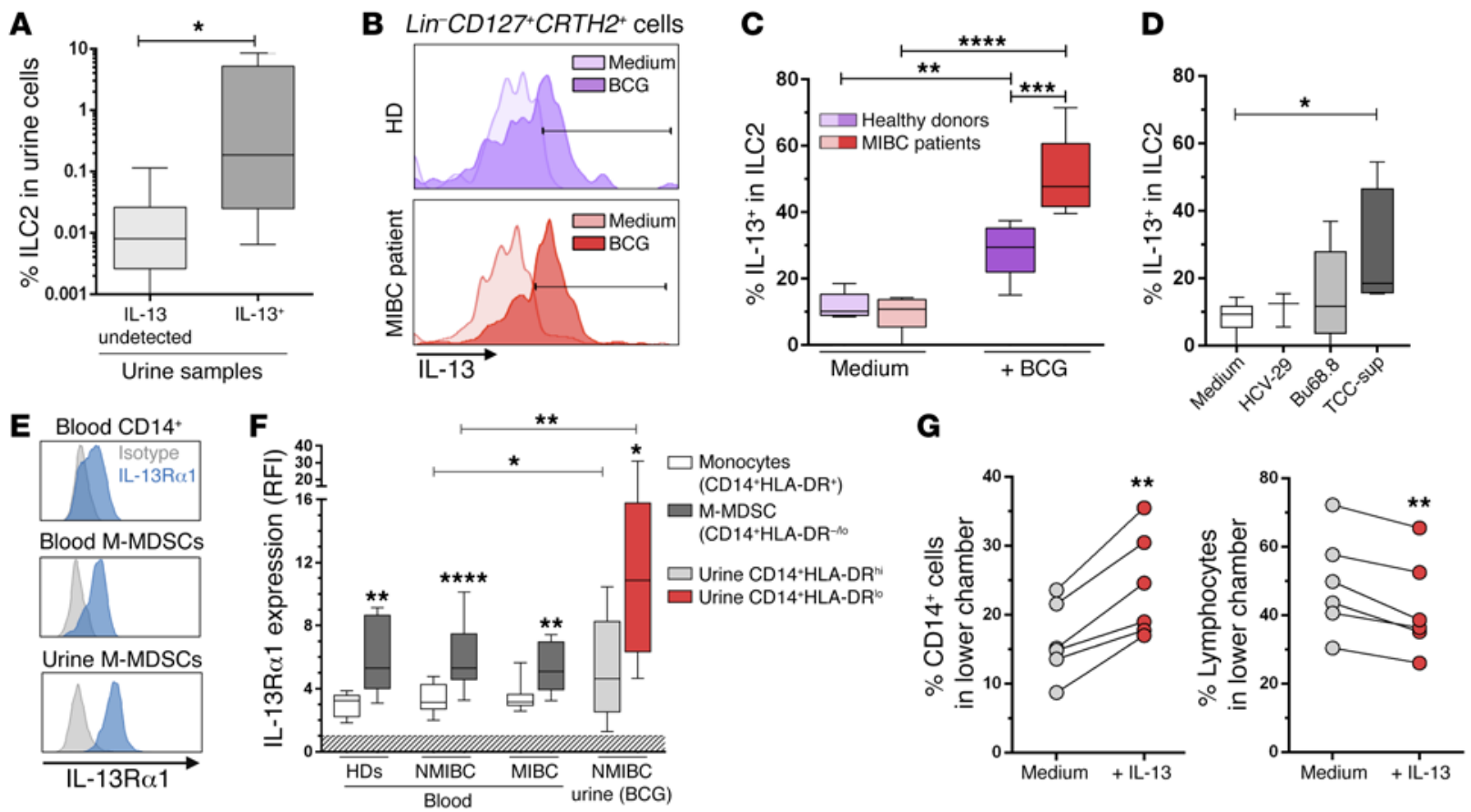

H
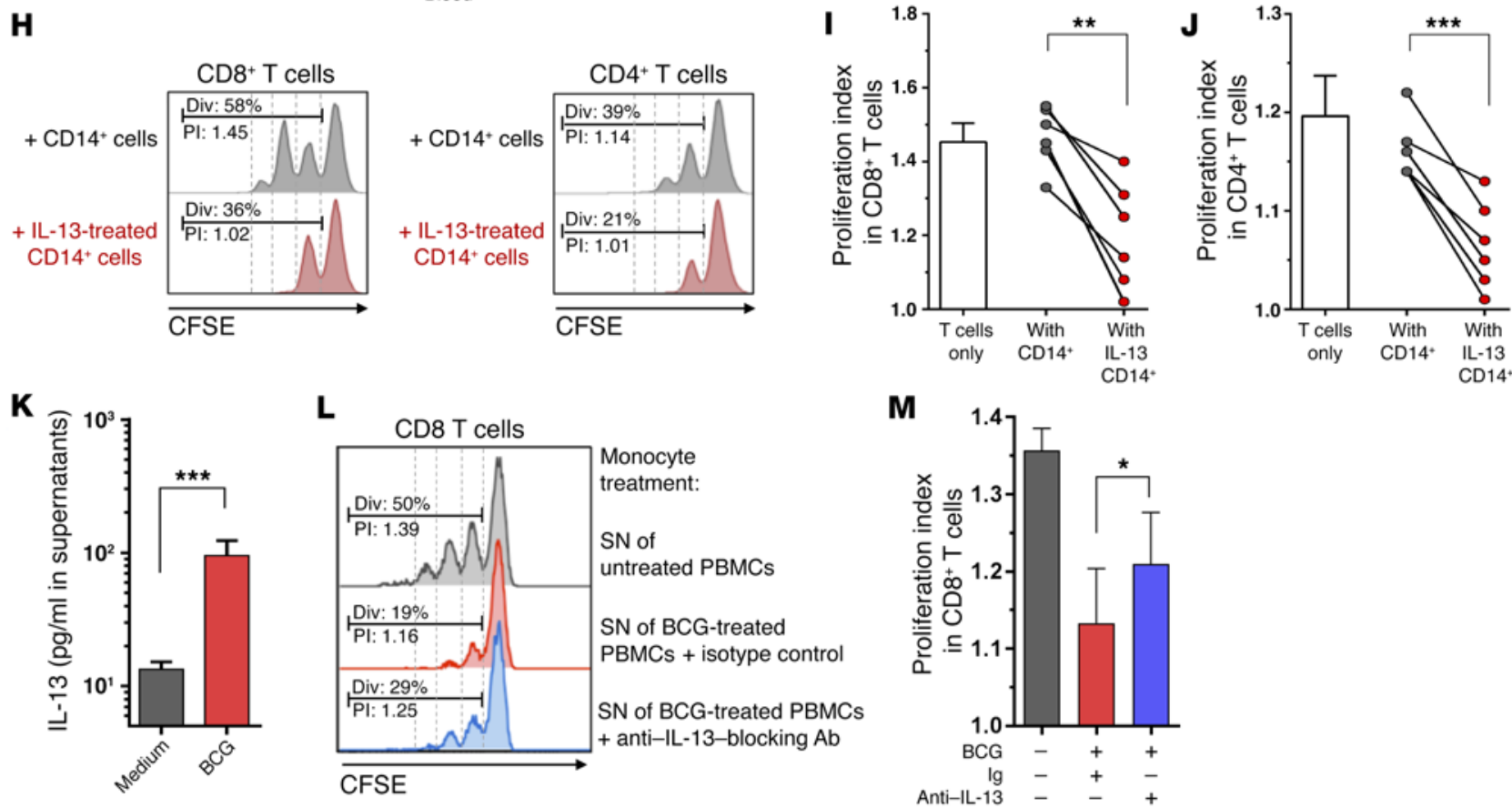

Figure 5. IL-13 is produced by ILC2 upon BCG stimulation and is able to recruit and induce suppressive function in monocytic cells. (A) Frequencies of ILC2 in urine samples with undetectable $(n=7)$ versus detectable $(n=6)$ urinary IL-13 (detection limit: 5 pg/ml). (B) Representative FACS histograms of IL-13-producing ILC2 upon stimulation with BCC $(\mathrm{MOI}=1)$ or medium alone in PBMCs from HDs $(n=6)$ and MIBC patients $(n=5)$ and $(C)$ their quantification. (D) IL-13 production in ILC2 from patients with MIBC following coculture with nonmalignant urothelium (HCV-29), Bu68.8, or TCC-Sup tumor cell lines. (C and D) Statistical analysis was performed using a 2-tailed, paired Student's $t$ test to compare stimulated versus medium-only conditions and a 2-tailed, unpaired Student's $t$ test to compare patients with HDs (C) and 1-way ANOVA followed by Dunnett's test to compare cell lines with the control condition (D). (E) Representative example of IL-13R $\alpha 1$ labeling. (F) Levels of ex vivo IL-13R $\alpha 1$ expression on the indicated monocytic cells from PBMCs obtained from HDs $(n=9)$, NMIBC patients $(n=18)$, and MIBC patients $(n=10)$ and from post-BCG urine samples $(n=8)$, expressed as the ratio of mean fluorescence intensity (RFI) of specific staining versus the isotype Ig control. A 2-tailed, paired Student's $t$ test was performed to compare M-MDSCs with CD14+HLA-DR ${ }^{+/ h i}$ cells and an unpaired Student's $t$ test to compare urine with circulating counterparts. (C) Chemotactic response of CD14+ cells and CD3+ $/$ CD19+ lymphocytes from HD PBMCs ( $n=6$; upper chamber) to medium alone or IL-13 (100 ng/ml; lower chamber) using Transwell plates. (H) Representative FACS histograms of CFSE-labeled activated T cells cultured alone or with purified CD14+ cells pretreated with IL-13 (IL-13-treated CD14+ cells) or not (CD14+ cells). (I and J) Quantification of the proliferation index in CD8 ${ }^{+}(\mathbf{I})$ and CD4+ (J) T cells. (K) IL-13 concentration in supernatants of PBMCs $(n=8)$ subjected to 3 days of stimulation with BCG or no stimulation. (L) Representative example of CFSE-labeled activated CD8 ${ }^{+} \mathrm{T}$ cells cocultured with $\mathrm{CD} 14^{+}$cells previously conditioned with filtered supernatants of unstimulated or BCC-stimulated PBMCs in the presence of either anti-IL-13-blocking antibody or isotype IgG1 control $(10 \mu \mathrm{g} / \mathrm{ml}$ each). (M) Comparison of the proliferation index in each indicated condition ( $n=3$, representative of 2 independent sets of experiments). ${ }^{*} P<0.05,{ }^{* *} P<0.01,{ }^{* *} P<0.001$, and ${ }^{* * *} P<0.0001$, by 2-tailed, paired Student's $t$ test (G-K) and 1-way ANOVA followed by Tukey's test (M). Div, percentage of cells with at least 1 division; Ig, isotype IgG1 control; PI, proliferation index; SN, supernatant. 
A
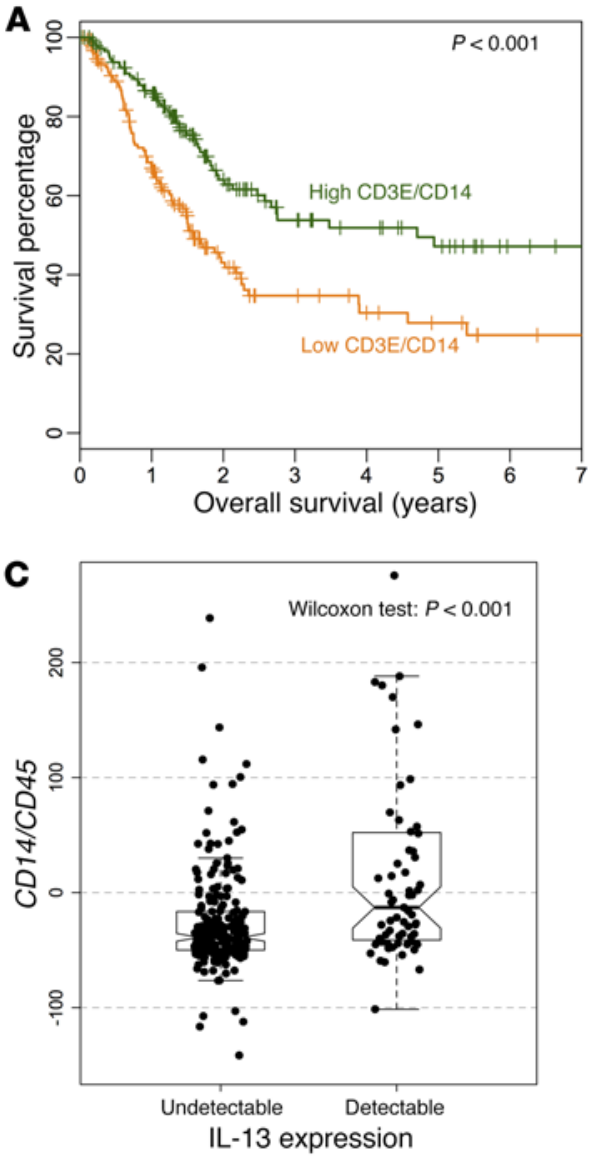

B

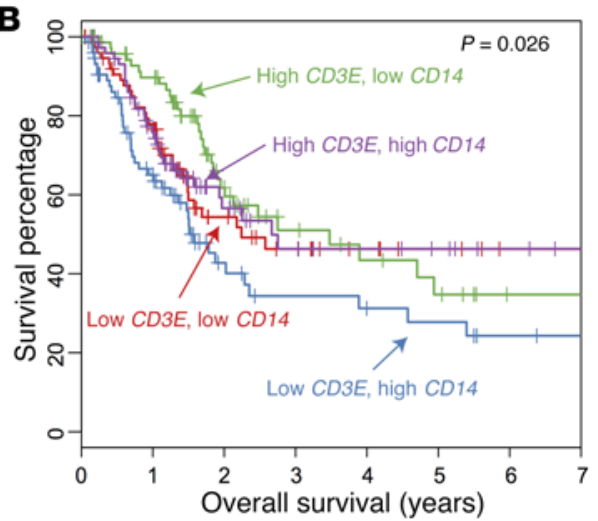

D

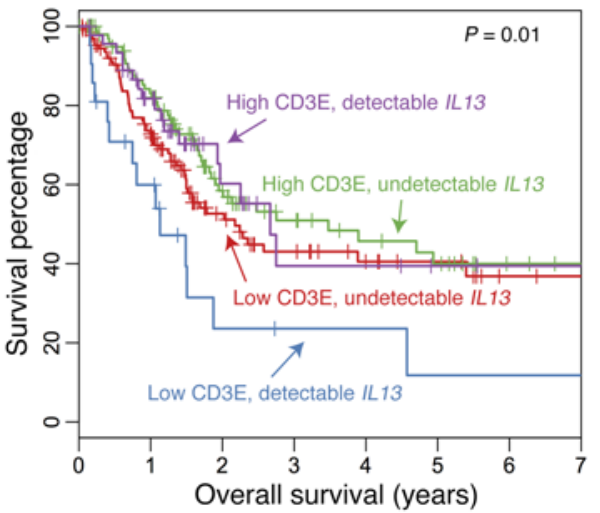

Figure 6. TCGA data analysis for MIBC. Tumor mRNA expression levels were obtained from TCGA for 300 patients with BCa. (A) Survival analysis based on the CD3E/CD14 expression ratio (the median value was used to split samples). (B) Patients were first segregated on the basis of their $C D 3$ levels (below and above the median value), and both groups were subsequently divided into 2 subgroups on the basis of CD14 levels (below and above the respective median values in the subgroups). (C) Comparison of CD14 levels (normalized as described in the Methods section) between samples with detectable and undetectable IL13 expression levels. (D) Survival analysis based on CD3E (the median value was used to split samples) and IL13 expression (detectable versus undetectable expression). $P$ values were determined by log-rank test (A, B, and D) and the Wilcoxon test (C). the tumor immune contexture. Specifically, ex vivo and in vitro data support the involvement of an ILC2/IL-13 axis in the recruitment and induction of M-MDSCs, thus pointing to a detrimental role for ILC2 in the context of cancer immunotherapy (Figure 7).

Since its FDA approval in 1990, intravesical BCG is one of the most successful cancer biotherapies to date, and yet the mechanisms involved in treatment success or failure remain poorly understood (8). The present study ascribes a critical role to the local balance between $\mathrm{T}$ lymphocytes and myeloid suppressor cells in the clinical response to BCG therapy, independent of patient and tumor characteristics (stage and grade) (Table 1). Indeed, a strong prognostic value with respect to recurrence was found when considering the T cell-to-MDSC ratio, instead of the levels of each individual cell subset. We can thus speculate that effective antitumor $\mathrm{T}$ cell activity may only be generated when $\mathrm{T}$ cells outnumber the locally recruited/induced M-MDSCs. Of note, the importance of the effector-to-regulatory balance was previously suggested in human cancers, but involved FoxP3 ${ }^{+}$Tregs instead of MDSCs (17, 33). These findings raise the possibility that the levels of $\mathrm{T}$ cells and M-MDSCs in urine after initiation of BCG therapy may be a useful predictive biomarker of therapeutic efficacy. However, the fact that (a) the patient dichotomy based on the T cell/MDSC ratio may be present before the start of the therapy (Figure 2E) and that (b) this ratio may be linked to MIBC patient survival (Figure 6) suggest a prognostic role of the $\mathrm{T}$ cell/MDSC ratio rather than a prediction of BCG treatment failure. Thus, further validation of this ratio's prognostic or predictive value in a larger cohort of patients is warranted.
While MDSCs have been extensively studied in mouse cancer models (10), the data are sparser with regard to their clinical prognostic value in human cancers (34). Most studies consistently found - mainly in peripheral blood - elevated granulocytic and/or monocytic MDSC levels in patients with various cancers, including $\mathrm{BCa}(35,36)$, and the levels were often associated with the tumor stage (34), which is in line with our peripheral MDSC data. In tissue, high infiltration of $\mathrm{CD} 68^{+}$tumor-associated macrophages (TAMs) was associated with a poor response to BCG immunotherapy (37). Notably, the beneficial versus deleterious role of the tumor-associated immune contexture (38) might depend on the localization of immune cell subsets, which can be associated with different clinical outcomes according to whether these subsets are assessed in the tumor core versus the invasive margin, or inside versus outside the tertiary lymphoid structures (39). Likewise, the level of $\mathrm{CD} 68^{+}$macrophages in bladder CIS was associated with a poor response to BCG therapy only when measured in the tumor, and not in the bladder lamina propria (40). However MDSCs, in contrast to T cells, cannot be accurately appraised in situ by IHC. This points out the unmet need to identify specific and sensitive markers able to reliably define MDSCs for in situ assessment.

The growing field of ILC research is still in its infancy for human cancers $(41,42)$, although some recent studies reported on ILCs in murine tumor models. ILC1, and potentially ILC3, may be involved in tumor immunosurveillance $(43,44)$. However, the role of ILC2 - otherwise involved in anti-helminth immunity and tis- 


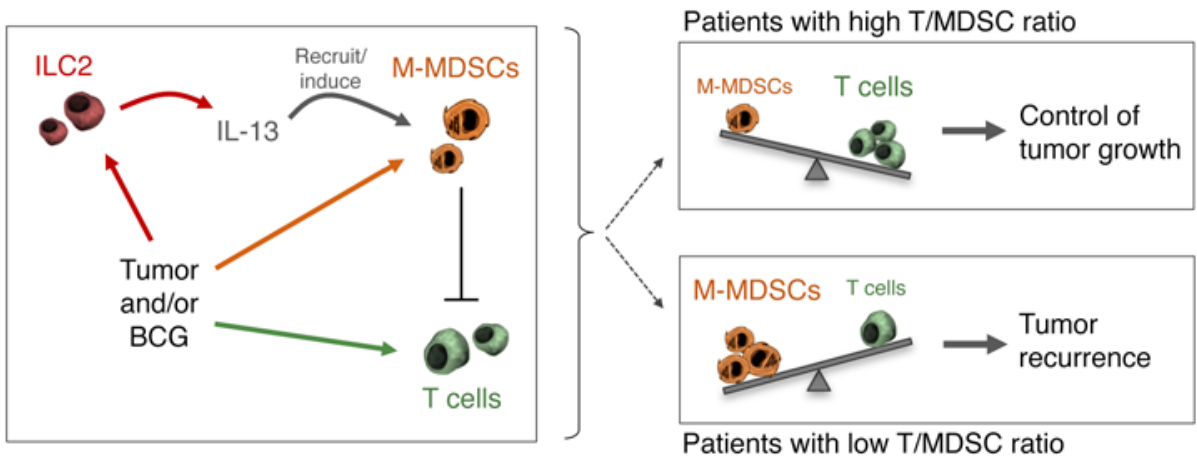

Figure 7. Putative model of a suppressive axis involved in BCa recurrence. Schematic representation of the immune profiles associated with a high or low risk of bladder tumor recurrence. Interactions between tumor cells or BCG and immune cell subsets that are proposed to play an important role in the disease outcome are indicated. Both tumor and BCG can directly or indirectly recruit MDSCs and T cells locally, as well as recruit and activate ILC2 to produce IL-13 that can amplify MDSC recruitment and induction. Finally, the resulting balance between MDSC and T cell levels may be a critical factor regulating tumor recurrence.

sue homeostasis - might be detrimental in the context of tumors, as recently suggested in a mouse cancer model (29). Interestingly, we identified ILCs both locally and in the periphery in patients with $\mathrm{BCa}$ and observed a skewing toward a group 2 ILC phenotype that was associated with a worsening of the disease. A recent study reported that murine tissue-resident ILC2 expand locally in inflammatory conditions (45). Accordingly, bladder tumors and intravesical BCG stimulation may favor ILC2 proliferation/ induction in the bladder, as supported by in vitro data. An important finding in our study was the positive correlation between ILC2 and M-MDSC levels, both locally during BCG therapy and in the blood of patients with MIBC. Our data suggest that IL-13 is a link between both cell subsets, as (a) M-MDSCs recruited to the bladder highly expressed the IL-13R $\alpha 1$; (b) ILC2 were prone to secrete IL-13 in response to BCG and tumor cells in vitro; (c) urine samples with detectable IL-13 corresponded to samples with higher ILC2 frequencies; and (d) IL-13 was found to both preferentially recruit and induce suppressive function in monocytic cells in vitro. IL-13 is a type 2 cytokine involved in antiparasite immunity, fibrosis, and asthma (46) and is known, like IL-4, to induce the so-called alternative activation of macrophages toward the M2 phenotype (28). M2-like TAMs and MDSCs are not clearly demarcated entities and thus exhibit many common characteristics, notably the ability to favor a tumor-supporting and immune-tolerogenic microenvironment (11). Several mouse studies documented the role of IL-13 in the upregulation of ARG1 (47), an enzyme that we found highly expressed in urine M-MDSCs and that was induced following IL-13 treatment in monocytic cells in vitro. Murine MDSCs are significantly more suppressive when cultured with IL-13, and this effect is attributed to ARG1 upregulation (48). Accordingly, human M-MDSCs from head and neck cancer patients were found to exert a suppressive function via ARG1 (49). Elevated IL-13 levels, concomitant with increased MDSC, M2, and/or ARG1 levels, were reported in human gastrointestinal cancers $(50,51)$. Moreover, an ILC2/IL-13/M2 axis was recently reported in mouse models of parasitic infections $(52,53)$ and of breast cancer $(29)$, supporting our results.
Both tumor cells and BCG may promote the ILC2/IL-13/MDSC axis. We showed that $\mathrm{BCa}$ cells are able to induce suppressive M-MDSCs in vitro, as was previously observed in different cell lines from various cancers (54). Thus, a basal immunosuppressive level may exist in the bladder before treatment, as supported by the correlation between early pre-BCG and late postBCG samples. Second, intravesical BCG stimulation triggers a substantial immune infiltration, including infiltration of M-MDSCs and ILC2, the composition of which may be the result of different events: modulation/proliferation of already locally present immune cells, recruitment from the periphery by urothelium-derived chemokines, and, in a second phase, modulation of the locally recruited cells. While mycobacteria are potent inducers of Th1 responses (55), which are required for BCG-mediated bladder tumor control (8), we found that BCG can also induce MDSCs, as shown in intradermally BCG-vaccinated mice (56). In addition, BCG shifted ILCs toward the ILC2 phenotype, at the expense of ILC1. A recent study supports this finding, reporting an increase in ILC2 levels in Mycobacterium leprae/HIV-coinfected patients compared with levels in HIV-monoinfected and healthy individuals (57). Further investigations may evaluate whether ILC2 skewing is due to ILC2 proliferation or repolarization from another ILC subset, as was recently suggested $(58,59)$.

While induction of ILC2 following mycobacteria infection/ instillation may drive tissue repair $(12,13)$, these cells may be also involved in the pathophysiology of diseases such as cancer, in which type 1 immunity is required. In $\mathrm{BCa}$, besides inducing a strong Th1 response, which may depend on preexisting BCGspecific adaptive immunity (60), BCG may also amplify a preestablished immunosuppressive tumor contexture containing MDSCs and ILC2. This needs to be compensated by a higher BCG-induced $\mathrm{T}$ cell infiltration, without which patients experience rapid tumor recurrence.

While immunotherapy using an anti-PD-L1 antibody was recently found to show clinical efficacy in advanced metastatic $\mathrm{BCa}$, with a breakthrough status designated by the FDA (61), new combination treatments are urgently needed for NMIBC to improve BCG therapy and lower BCG failure rates (62). This study highlights the crucial need to restrain the tumor-induced "immunosuppressive switch" (63) by targeting MDSCs to tilt the balance in favor of T cells. A number of compounds were found to target MDSCs (10, 11). One of these compounds, doxorubicin, may be of interest, as it selectively targets monocytic MDSCs in humans (64). However, in light of our results, all-trans retinoic acid (ATRA, also known as vitamin A) may be an ideal candidate treatment on the basis of the following properties: (a) this compound is also known to eliminate MDSCs and thus to augment antigen-specific $\mathrm{T}$ cell responses (65), including in cancer patients (66); (b) Spencer et al. recently reported that vitamin A strongly restrains ILC2 levels, particularly 
ILC2 that produce IL-13 (67); and (c) a meta-analysis showed that vitamin A plays a protective role in BCa (68), and ATRA treatment was found to significantly improve survival and recurrence rates in NMIBC patients (69). Furthermore, a combination of depletion or attenuation of MDSCs and concomitant stimulation of effector $\mathrm{T}$ cells may be of interest. In this regard, programmed death 1 (PD-1) and cytotoxic T lymphocyte-associated protein 4 (CTLA-4) combination blockade has proven to increase the T cell-to-MDSC ratio in melanoma tumors in mice (18).

The present study highlights the importance of the immune microenvironment in the clinical outcome of BCG-treated superficial BCa and suggests a possible new role for ILC2 in human cancer. We propose that the ILC2/IL-13 axis is a mechanism driving an immunosuppressive microenvironment that is critically involved in the failure of BCG immunotherapy (Figure 7). Such a microenvironment could also influence the mortality of patients with more advanced cancer, as suggested by our analysis of BCa data from TCGA. Altogether, these results imply that M-MDSCs and novel pathways, such as IL-13/IL-13R signaling or ILC polarization, may represent attractive targets for potentiating cancer immunotherapies.

\section{Methods}

Study populations. Twenty-eight patients with NMIBC were recruited in this prospective, longitudinal study at the University of Lausanne Hospital. At the time of inclusion, patients started standard BCG therapy involving 6 weekly intravesical instillations of TICE BCG (MSD: Merck Sharp \& Dohme AG) $\left(2 \times 10^{8}\right.$ to $\left.8 \times 10^{8} \mathrm{CFU}\right)$, according to European Association of Urology guidelines and to the decision of both physicians and patients. NMIBC patients' characteristics are described in Table 1. Urine samples were obtained before and 4 hours ( \pm 1 hour) after each instillation. Peripheral blood was also collected at baseline and during week 4 . In addition, peripheral blood from 18 patients with MIBC was collected at the time of cystectomy. MIBC patients' characteristics are described in Supplemental Table 1. Blood samples were also collected from healthy volunteers ( $>50$ years of age) through the local Swiss blood bank.

Clinical follow-up. Following BCG therapy, flexible cystoscopy and cytology were performed on a regular basis. In instances of positive cytology results and/or a visible tumor, subsequent transurethral resection of the bladder (TURB) allowed confirmation of the presence or absence of a recurrent tumor. Tumor progression was defined as the recurrence of a tumor at a higher stage or grade and/or worsening of disease, as defined in refs. 6 and 70.

Flow cytometric analysis of urine-infiltrating immune cells. Urine samples were centrifuged to separate cells from the urine supernatant. The total number of cells in urine was independent of the voided volume (our unpublished observations). The following mAbs were used at predetermined optimal concentrations: anti-CD45-phycoerythrin (PE) (2D1); anti-CD11b-allophycocyanin (APC)/eFluor 780 (ICRF44); anti-CD3-PerCP/Cy5.5 (SK7); anti-FceR1 $\alpha$-FITC (AER-37, CRA1); antiCD3-PE/Cy7 (UCHT1); anti-CD14-PE/Cy7 (61D3); anti-CD19-PE/Cy7 (SJ25C1); anti-CD303a-PE/Cy7 (201A) (all from eBioscience); antiCD14-Pacific Blue (HCD14); anti-CD15-PerCP/Cy5.5 (W6D3); antiCD33-APC (WM53); anti-HLA-DR-PE/Cy7 (L243); anti-CD15-Pacific Blue (W6D3); anti-CD56-PE/Cy7 (HCD56); anti-CD117 (c-Kit)-PE (104D2); anti-CD294(CRTH2)-PerCP/Cy5.5 (BM16); anti-CD11c-PE/
Cy7 (Bu15); anti-CD15-PE/Cy7 (W6D3); anti-CD163-PE (GHI/61); anti-CD64-PerCP/Cy5.5 (10.1); and anti-CD68-AF647 (Y1/82A) (all from BioLegend); anti-CD3-PE/AF610 (7D6); anti-CD19-PE/AF610 (SJ25C1); anti-CD56-PE/Texas Red (MEM-188); and anti-CD8-PE/ AF610 (3B5) (all from Invitrogen, Thermo Fisher Scientific); anti-CD4APC/H7 (RPA-T4); anti-CD127-AF647 (HIL-7R-M21); anti-CD203cBV421 (NP4D6); anti-CD80-APC/H7 (L307); anti-CD115(CSF-1R)BB515 (9-4D2); and anti-CCR4-PE/Cy7 (1G1) (all from BD Biosciences); and anti-IL-13R $\alpha 1-P E$ (419718) (from R\&D Systems).

Cells were stained for surface antigens for 20 minutes at $4^{\circ} \mathrm{C}$, and an amine reactive dye (Aqua LIVE/DEAD Stain Kit; Life Technologies, Thermo Fisher Scientific) was used for dead cell exclusion according to the manufacturer's instructions. Fc-Receptor Blocking Reagent (Miltenyi Biotec) was used to increase staining specificity by blocking unwanted binding of antibodies. Sample acquisition was performed on the Gallios Flow Cytometer (Beckman Coulter), and data were analyzed using FlowJo software.

Intracellular cytokine staining. Cells were stimulated with BCG $(\mathrm{MOI}=1)$ for 48 hours, and $2.5 \mu \mathrm{g} / \mathrm{ml}$ brefeldin A was added for the last 16 hours of culture. Staining for cell-surface markers was carried out as described above, and cells were then fixed. Intracellular staining was performed with APC-conjugated anti-IL-13 mAb (JES10-5A2; BD Pharmingen) for 30 minutes at $4^{\circ} \mathrm{C}$ in $0.1 \%$ saponin (Sigma-Aldrich).

Cell lines and bacteria. Bu68.8 is a noninvasive BCa cell line isolated from a patient with a TaG2 tumor at the urology department of the University of Lausanne Hospital. T24 and TCC-Sup are invasive urothelium carcinoma cell lines corresponding to histological grades G3 and G4, respectively (71) (gift of G.N. Thalmann, Inselspital, Bern, Switzerland). HCV-29 is a nonmalignant cell line derived from normal urothelium (71) (gift of Anna Lityńska, Jagiellonian University, Kraków, Poland). For the cocultures, tumor cells were seeded to achieve confluence by the end of the experiment as determined beforehand (e.g., a ratio of approximately 1:20 with PBMCs in 4-day-long experiments).

Bacteria used for in vitro experiments were obtained by resuspension in PBS of the lyophilized content of clinically available oncoTICE BCG vials and Vivotif Ty21a capsules.

Suppression assays. Monocyte isolation was performed using the Dynabeads FlowComp Human CD14 Kit (Invitrogen) from cryopreserved PBMC or as previously described (72) from fresh blood $(>85 \%$ purity). CD14 ${ }^{+}$cells were cultured for 4 days with $400 \mathrm{U} / \mathrm{ml} \mathrm{GM-CSF}$ (ImmunoTools) in the presence or absence of $100 \mathrm{ng} / \mathrm{ml}$ recombinant human IL-13 (BioLegend), or with filtered supernatant (70 \%) from PBMCs stimulated or not for 72 hours with BCG $(\mathrm{MOI}=1)$. When indicated, anti-IL-13-neutralizing antibody or the corresponding IgG1 isotype control $(10 \mu \mathrm{g} / \mathrm{ml}$; BioLegend) was added. Autologous T cells were isolated from PBMCs using the Dynabeads FlowComp Human CD3 Kit (Invitrogen, Thermo Fisher Scientific), stained using $5 \mu \mathrm{M}$ CFSE for 5 minutes at room temperature, and washed twice with icecold PBS containing 5\% FCS. CD $14^{+}$cells were cocultured for 4 days with CFSE-labeled $\mathrm{T}$ cells at a 1:1 ratio in 96-well pates previously coated with $1 \mu \mathrm{g} / \mathrm{ml}$ anti-CD3 (OKT3) antibody and soluble anti-CD28 (Biolegend) $(1 \mu \mathrm{g} / \mathrm{ml})$ and IL-2 (150 U/ml; Roche). Proliferation of CFSE-labeled cells was assessed by flow cytometry after surface staining for CD3, CD4, and CD8. The proliferation index (total number of divisions divided by the number of cells that went into division) was assessed using the FlowJo Proliferation Platform. 
Sorting of urine cell subsets, cDNA synthesis, and real-time qPCR. $\mathrm{Lin}^{-} \mathrm{CD} 14^{+} \mathrm{CD} 33^{+} \mathrm{HLA}-\mathrm{DR}{ }^{\text {lo }}$ and $\mathrm{Lin}^{-} \mathrm{CD} 14^{+} \mathrm{CD} 33^{+} \mathrm{HLA}-\mathrm{DR} \mathrm{R}^{\text {hi }}$ cells were FACS sorted from post-BCG urine samples and subsequently tested for purity (Supplemental Figure 1). Five samples from patients included in the present cohort as well as two samples from patients receiving SII-ONCO-BCG at the University Hospital of Basel were tested. Dry pellets (900-10,000 cells) were then resuspended in a lysiscDNA mix solution containing $6.3 \mu$ lysis buffer (prepared as previously described by Gupta et al. [ref. 73]); $3 \mu \mathrm{l} 5 \times$ reverse transcriptase (RT) buffer (73); $1.5 \mu \mathrm{l}$ of 0.1 M DTT (AppliChem); $0.75 \mu \mathrm{l}$ of $10 \mathrm{mM}$ deoxyribonucleotide triphosphates (dNTPs) (Invitrogen, Thermo Fisher Scientific); $0.25 \mu \mathrm{l}$ of $100 \mathrm{ng} / \mu \mathrm{l}$ oligo-(dT) (Metabion); $0.4 \mu \mathrm{l}$ Moloney Murine Leukemia Virus (M-MLV) Reverse Transcriptase (Invitrogen, Thermo Fisher Scientific); $0.2 \mu \mathrm{l}$ RNasin (Promega); and $2.6 \mu \mathrm{l}$ RNAse-free water. To allow reverse transcription into cDNA, samples were incubated at $37^{\circ} \mathrm{C}$ for 60 minutes. The transcriptase was then inactivated at $90^{\circ} \mathrm{C}$ for 3 minutes, and samples were stored at $-80^{\circ} \mathrm{C}$. Quantitative real-time PCR was performed using KAPA SYBR FAST Master Mix with ROX (KAPA Biosystems), $200 \mathrm{nM}$ of each primer, and one-twentieth of the reverse transcription reaction. Samples were run at least in duplicate in an ABI 7500 Fast Real-Time PCR Thermo Cycler (Applied Biosystems) with the following parameters: 3 minutes at $95^{\circ} \mathrm{C}$ for enzyme activation, 40 cycles at $95^{\circ} \mathrm{C}$ for 5 seconds and $60^{\circ} \mathrm{C}$ for 30 seconds, followed by amplicon melting analysis to evaluate the specificity of the reaction and identify the presence of primer dimers. B2-microglobulin (B2M) was used as the reference gene, as it was previously identified as one of the most stable genes in activated monocytic cells (74). Primers were purchased from Microsynth, and efficiency was validated using cDNA serial dilutions. The primers sequences were as follows: B2M, 5'-CCAGCAGAGAATGGAAAG-3' (forward) and 5'-GATGCTGCTTACATGTCT-3' (reverse); ARG1: 5'-ATTCTTCCGTTCTTCTTGACTT-3' (forward) and 5'-AGTGTGATGTGAAGGATTATG-3' (reverse); INOS: 5'-ATGCTCAGCTCATCCGCTAT-3' (forward) and 5'-CACAAGGTCAGGTGGGATTT-3' (reverse); and CEBPB: 5'-GCCGGTTTCGAAGTTGAT-3' (forward) and 5'-CAACAAGCCCGTAGGAACAT-3' (reverse). Results are expressed using the $2^{-\Delta \Delta C t}$ method.

Measurement of soluble molecules. Cell-free urine samples were stored at $-80^{\circ} \mathrm{C}$. Cytokines were measured using a Luminex assay according to the manufacturer's instructions (Thermo Fisher Scientific), and tryptophan and kynurenine levels were measured by Metabolon Inc. using ultra-high-performance liquid chromatography-tandem mass spectroscopy (UPLC-MS/MS) (75). The LC/MS portion of the platform was based on a Waters ACQUITY UPLC and a Thermo Scientific Q-Exactive high-resolution/accurate mass spectrometer. Peaks were quantified using the AUC, and relative kynurenine levels were divided by those of tryptophan to assess the $\mathrm{K} / \mathrm{T}$ ratio in each sample.

Ex vivo-expanded urine $T$ cells. Cells from urine samples were stimulated, either directly or following magnetic T cell enrichment, with PHA $(1 \mu \mathrm{g} / \mathrm{ml})$ and IL-2 $(150 \mathrm{U} / \mathrm{ml})$ in the presence of irradiated allogenic PBMCs for 2 to 4 weeks, as we previously described (76). When $\mathrm{T}$ cells successfully expanded, $\mathrm{CD} 4^{+} \mathrm{T}$ cells were isolated using the Dynabeads FlowComp Human CD4 Kit (Invitrogen, Thermo Fisher Scientific) and stained for Th1 and Th2 surface markers. The remaining CD4 cells were stimulated with immobilized anti-CD3 and soluble anti-CD28 for 24 hours, and the supernatant was collected to measure IFN- $\gamma$, IL-2, TNF- $\alpha$, IL- 4 , and IL- 5 cytokines using a
Cytometric Bead Array (BD Biosciences). For each sample, the concentration [i] for the cytokine i was normalized to the maximum concentration $[\mathrm{i}]_{\max }$ observed for this cytokine (Equation 1), and the Th2 versus Th1 score (Equation 2) was determined. These equations were calculated as follows:

$$
C_{i}=\frac{[i]}{[i]_{\max }} \times 100
$$

(Equation 1)

$$
\text { Score }=\overline{C_{T h 2}}-\overline{C_{T h 1}}=\frac{1}{2} C_{I L-4}+\frac{1}{2} C_{I L-5}-\frac{1}{3} C_{I F N-\gamma}-\frac{1}{3} C_{L L-2}-\frac{1}{3} C_{T N F-\alpha}
$$

\section{(Equation 2)}

Data and computational analysis in TCGA. Gene expression data for bladder urothelial cancer from TCGA (32) were obtained through release 18 from the International Cancer Genome Consortium (ICGC; https://icgc.org/). Gene expression was normalized to counts per million before analysis. Survival data for the same patients were obtained with the FirebrowseR package in R (https:/github.com/mariodeng/ FirebrowseR). We used the R survival package to plot the KaplanMeier curves and estimate the log-rank difference between the various groups of donors studied. The analysis was done on the overall survival of the donors. As IL-13 was not detectable in all samples, we defined a threshold, assuming IL13 was present in the sample when more than 2 reads mapped to its gene.

Computation of excess CD14. The level of infiltration of the different immune cells is in general highly correlated, such that we could model at first approximation the expression of CD14 as:

$$
\mathrm{CD} 14=\alpha \times \mathrm{CD} 45+\beta
$$

(Equation 3)

To study the effect of IL13 on CD14, it is important to estimate how much the expression of CD14 differs from the expected value coming from the global immune infiltrate. Using all the samples, we estimated the coefficients $\alpha$ and $\beta$ from Equation 3. Then, for each patient, we computed the residue of this fit: a positive residue corresponded to an excess of CD14 with respect to the expected amount, while a negative value represented a deficiency in CD14. The residues are shown in Figure 6C.

Statistics. Comparisons between samples and individuals or between conditions in the same individuals were evaluated using an unpaired or paired (2-tailed) Student's $t$ test, respectively, directly or after log transformation to meet normality assumptions. For multiple comparison analyses, 1-way ANOVA (for paired or unpaired values as appropriate) was performed, followed by Dunnett's correction when all data were compared with a control condition, by Tukey's correction when all data were compared, one with another, or by a post test for linear trend to assess longitudinal data. A P value of less than 0.05 was considered statistically significant. The Kaplan-Meier estimator was used to assess recurrence- and progression-free survival rates. The log-rank test was performed to analyze potential differences in survival rates between groups. The median follow-up time was calculated by using the reverse Kaplan-Meier method (77). Comparisons of patients' 
characteristics were performed using a Fisher's exact or $\chi^{2}$ test. All statistical analyses were performed using R or GraphPad Prism, version 6 (GraphPad Software).

Study approval. This study was approved by the ethics committee of the canton of Vaud, Switzerland. Some urine samples were obtained from patients receiving SII-ONCO-BCG at the University Hospital of Basel with the approval of the Basel ethics committee (protocol EKBB161/09). All patients provided written informed consent prior to their participation in the study.

\section{Author contributions}

MFC, ST, CJ, and LD designed the study and wrote the manuscript. MFC, ST, BS, VC, D. Gharbi, PB, and SDP performed and analyzed the experiments. JR and D. Gfeller conducted in silico analyses. MFC, ST, JR, and LD performed statistical analyses. JR, DNH, CAR, DES, D. Gfeller, and PJ provided intellectual input and edited the manuscript. FD, ASF, CAR, and PJ recruited the study participants and provided patients' samples and clinical data. CJ and LD jointly supervised the study.

\section{Acknowledgments}

We are obliged to the patients and blood donors for their dedicated collaboration. We thank Pedro Romero for the fruitful discussions and kind support of this study. We are grateful to G.N. Thalmann (Inselspital, Bern, Switzerland) and Anna Lityńska (Jagiellonian University, Kraków, Poland) for providing bladder cell lines. This work was supported by grants from the Swiss National Foundation (32003B_146638, to LD); the Novartis Foundation for MedicalBiological Research (15C165, to LD); the Foundation for the Fight Against Cancer (369 and 324, to LD); the Swiss National Foundation Ambizione (PZOOP3_161459, to CJ); the Fondazione San Salvatore (to CJ); ProFemmes UNIL (to CJ); the Fondation Pierre Mercier pour la Science (to CJ); the Swiss Cancer League (KFS3710-08-2015-R, to CJ); and the Marie Heim Vögtlin Fellowship of the Swiss National Foundation (PMPDP3_164447, to ST).

Address correspondence to: Laurent Derré, CHUV BU48/510, Rue du bugnon, 48, 1011 Lausanne, Switzerland. Phone: 41.79. 556.3380; Email: laurent.derre@chuv.ch.
1. Gajewski TF, Schreiber H, Fu YX. Innate and adaptive immune cells in the tumor microenvironment. Nat Immunol. 2013;14(10):1014-1022.

2. Joyce JA, Fearon DT. T cell exclusion, immune privilege, and the tumor microenvironment. Science. 2015;348(6230):74-80.

3. Siegel RL, Miller KD, Jemal A. Cancer statistics, 2016. CA Cancer J Clin. 2016;66(1):7-30.

4. Messing EM. Why should we increase public awareness of bladder cancer, and how can we do it? Nat Clin Pract Urol. 2008;5(3):117.

5. Babjuk M, et al. EAU Guidelines on non-muscleinvasive urothelial carcinoma of the bladder: update 2016. Eur Urol. 2017;71(3):447-461.

6. Kamat AM, et al. Definitions, end points, and clinical trial designs for non-muscle-invasive bladder cancer: recommendations from the International Bladder Cancer Group. J Clin Oncol. 2016;34(16):1935-1944.

7. Nightingale SL. From the Food and Drug Administration. JAMA. 1990;264(6):677.

8. Redelman-Sidi G, Glickman MS, Bochner BH. The mechanism of action of BCG therapy for bladder cancer - a current perspective. Nat Rev Urol. 2014;11(3):153-162.

9. Bisiaux A, et al. Molecular analyte profiling of the early events and tissue conditioning following intravesical bacillus Calmette-Guérin therapy in patients with superficial bladder cancer.J Urol. 2009;181(4):1571-1580.

10. Marvel D, Gabrilovich DI. Myeloid-derived suppressor cells in the tumor microenvironment: expect the unexpected. J Clin Invest. 2015;125(9):3356-3364.

11. Ugel S, De Sanctis F, Mandruzzato S, Bronte V. Tumor-induced myeloid deviation: when myeloid-derived suppressor cells meet tumor-associated macrophages. JClin Invest. 2015;125(9):3365-3376.

12. Eberl G, Colonna M, Di Santo JP, McKenzie AN. Innate lymphoid cells. Innate lymphoid cells: a new paradigm in immunology. Science. 2015;348(6237):aaa6566.
13. Klose CS, Artis D. Innate lymphoid cells as regulators of immunity, inflammation and tissue homeostasis. Nat Immunol. 2016;17(7):765-774.

14. Marigo I, et al. Tumor-induced tolerance and immune suppression depend on the $\mathrm{C} / \mathrm{EBP}$ beta transcription factor. Immunity. 2010;32(6):790-802.

15. Domingos-Pereira S, Cesson V, Chevalier MF, Derré L, Jichlinski P, Nardelli-Haefliger D. Preclinical efficacy and safety of the Ty21a vaccine strain for intravesical immunotherapy of non-muscle-invasive bladder cancer. Oncoimmunology. 2017;6(1):e1265720.

16. Rentsch CA, et al. Bacillus Calmette-Guérin strain differences have an impact on clinical outcome in bladder cancer immunotherapy. Eur Urol. 2014;66(4):677-688.

17. Gao Q, et al. Intratumoral balance of regulatory and cytotoxic $\mathrm{T}$ cells is associated with prognosis of hepatocellular carcinoma after resection. JClin Oncol. 2007;25(18):2586-2593.

18. Curran MA, Montalvo W, Yagita H, Allison JP. PD-1 and CTLA-4 combination blockade expands infiltrating $T$ cells and reduces regulatory $\mathrm{T}$ and myeloid cells within $\mathrm{B} 16$ melanoma tumors. Proc Natl Acad Sci U S A. 2010;107(9):4275-4280.

19. Platten M, et al. Treatment of autoimmune neuroinflammation with a synthetic tryptophan metabolite. Science. 2005;310(5749):850-855.

20. $\mathrm{Xu} \mathrm{H}$, et al. Indoleamine 2,3-dioxygenase in lung dendritic cells promotes Th2 responses and allergic inflammation. Proc Natl Acad Sci US A. 2008;105(18):6690-6695.

21. Roan F, et al. $\mathrm{CD}^{+}{ }^{+}$group 1 innate lymphoid cells (ILC) form a functionally distinct ILC subset that is increased in systemic sclerosis. J Immunol. 2016;196(5):2051-2062.

22. Muthuswamy R, Wang L, Pitteroff J, Gingrich JR, Kalinski P. Combination of IFN $\alpha$ and poly-I:C reprograms bladder cancer microenvironment for enhanced CTL attraction. J Immunother Cancer. 2015;3:6.
23. Luo Y, Chen X, O'Donnell MA. Mycobacterium bovis bacillus Calmette-Guérin (BCG) induces human CC- and CXC-chemokines in vitro and in vivo. Clin Exp Immunol. 2007;147(2):370-378.

24. Yamada H, Luo Y, Matsumoto T, O’Donnell MA. A novel expression of macrophage derived chemokine in human bladder cancer. JUrol. 2005;173(3):990-995.

25. Reale M, et al. Production of MCP-1 and RANTES in bladder cancer patients after bacillus Calmette-Guerin immunotherapy. Cancer Immunol Immunother. 2002;51(2):91-98.

26. Xue L, et al. Prostaglandin D2 activates group 2 innate lymphoid cells through chemoattractant receptor-homologous molecule expressed on TH2 cells. J Allergy Clin Immunol. 2014;133(4):1184-1194.

27. Wojno ED, et al. The prostaglandin $\mathrm{D}_{2}$ receptor CRTH2 regulates accumulation of group 2 innate lymphoid cells in the inflamed lung. Mucosal Immunol. 2015;8(6):1313-1323.

28. Van Dyken SJ, Locksley RM. Interleukin-4- and interleukin-13-mediated alternatively activated macrophages: roles in homeostasis and disease. Annu Rev Immunol. 2013;31:317-343.

29. Jovanovic IP, et al. Interleukin-33/ST2 axis promotes breast cancer growth and metastases by facilitating intratumoral accumulation of immunosuppressive and innate lymphoid cells. Int J Cancer. 2014;134(7):1669-1682.

30. Magazin M, Guillemot JC, Vita N, Ferrara P. Interleukin-13 is a monocyte chemoattractant. Eur Cytokine Netw. 1994;5(4):397-400.

31. Thalmann GN, Sermier A, Rentsch C, Möhrle K, Cecchini MG, Studer UE. Urinary Interleukin-8 and 18 predict the response of superficial bladder cancer to intravesical therapy with bacillus Calmette-Guerin. JUrol. 2000;164(6):2129-2133.

32. Cancer Genome Atlas Research Network. Comprehensive molecular characterization of urothelial bladder carcinoma. Nature. 2014;507(7492):315-322.

33. Sinicrope FA, Rego RL, Ansell SM, Knutson KL, 
Foster NR, Sargent DJ. Intraepithelial effector $\left(\mathrm{CD}^{+}\right)$/regulatory $\left(\mathrm{FoxP}^{+}\right) \mathrm{T}$-cell ratio predicts a clinical outcome of human colon carcinoma. Gastroenterology. 2009;137(4):1270-1279.

34. Solito S, Marigo I, Pinton L, Damuzzo V, Mandruzzato S, Bronte V. Myeloid-derived suppressor cell heterogeneity in human cancers. Ann N Y Acad Sci. 2014;1319:47-65.

35. Yuan XK, Zhao XK, Xia YC, Zhu X, Xiao P. Increased circulating immunosuppressive CD14(+)HLA-DR(-/low) cells correlate with clinical cancer stage and pathological grade in patients with bladder carcinoma. J Int Med Res. 2011;39(4):1381-1391.

36. Brandau S, et al. Myeloid-derived suppressor cells in the peripheral blood of cancer patients contain a subset of immature neutrophils with impaired migratory properties. JLeukoc Biol. 2011;89(2):311-317.

37. Ayari C, et al. Bladder tumor infiltrating mature dendritic cells and macrophages as predictors of response to bacillus Calmette-Guérin immunotherapy. Eur Urol. 2009;55(6):1386-1395.

38. Fridman WH, Pagès F, Sautès-Fridman C, Galon $\mathrm{J}$. The immune contexture in human tumours: impact on clinical outcome. Nat Rev Cancer. 2012;12(4):298-306.

39. Giraldo NA, et al. Orchestration and prognostic significance of immune checkpoints in the microenvironment of primary and metastatic renal cell cancer. Clin Cancer Res. 2015;21(13):3031-3040.

40. Takayama H, et al. Increased infiltration of tumor associated macrophages is associated with poor prognosis of bladder carcinoma in situ after intravesical bacillus Calmette-Guerin instillation. JUrol. 2009;181(4):1894-1900.

41. Trabanelli S, et al. CD $127^{+}$innate lymphoid cells are dysregulated in treatment naïve acute myeloid leukemia patients at diagnosis. Haematologica. 2015;100(7):e257-e260.

42. Bie Q, et al. Polarization of ILC2s in peripheral blood might contribute to immunosuppressive microenvironment in patients with gastric cancer. J Immunol Res. 2014;2014:923135.

43. Dadi S, et al. Cancer immunosurveillance by tissue-resident innate lymphoid cells and innatelike T cells. Cell. 2016;164(3):365-377.

44. Carrega P, et al. NCR(+)ILC3 concentrate in human lung cancer and associate with intratumoral lymphoid structures. Nat Commun. 2015;6:8280.

45. Moro K, et al. Interferon and IL-27 antagonize the function of group 2 innate lymphoid cells and type 2 innate immune responses. Nat Immunol. 2016;17(1):76-86.

46. Wynn TA. Type 2 cytokines: mechanisms and therapeutic strategies. Nat Rev Immunol. 2015;15(5):271-282.

47. Gabrilovich DI, Nagaraj S. Myeloid-derived suppressor cells as regulators of the immune system. Nat Rev Immunol. 2009;9(3):162-174.

48. Highfill SL, et al. Bone marrow myeloid-derived suppressor cells (MDSCs) inhibit graft-versus- host disease (GVHD) via an arginase-1-dependent mechanism that is up-regulated by interleukin-13. Blood. 2010;116(25):5738-5747.

49. Vasquez-Dunddel D, et al. STAT3 regulates arginase-I in myeloid-derived suppressor cells from cancer patients. J Clin Invest. 2013;123(4):1580-1589.

50. Gabitass RF, Annels NE, Stocken DD, Pandha HA, Middleton GW. Elevated myeloid-derived suppressor cells in pancreatic, esophageal and gastric cancer are an independent prognostic factor and are associated with significant elevation of the Th2 cytokine interleukin-13. Cancer Immunol Immunother. 2011;60(10):1419-1430.

51. Gao J, et al. Infiltration of alternatively activated macrophages in cancer tissue is associated with MDSC and Th2 polarization in patients with esophageal cancer. PLOS ONE. 2014;9(8):e104453.

52. Besnard AG, et al. IL-33-mediated protection against experimental cerebral malaria is linked to induction of type 2 innate lymphoid cells, M2 macrophages and regulatory T cells. PLoS Pathog. 2015;11(2):e1004607.

53. Bouchery T, et al. ILC2s and T cells cooperate to ensure maintenance of M2 macrophages for lung immunity against hookworms. Nat Commun 2015;6:6970.

54. Lechner MG, et al. Functional characterization of human Cd33+ and Cd11b+ myeloid-derived suppressor cell subsets induced from peripheral blood mononuclear cells co-cultured with a diverse set of human tumor cell lines. J Transl Med. 2011;9:90.

55. Flynn JL, Chan J. Immunology of tuberculosis. Annu Rev Immunol. 2001;19:93-129.

56. Martino A, et al. Mycobacterium bovis bacillus Calmette-Guérin vaccination mobilizes innate myeloid-derived suppressor cells restraining in vivo T cell priming via IL-1R-dependent nitric oxide production. JImmunol. 2010;184(4):2038-2047.

57. Papotto PH, et al. New players in the same old game: disturbance of group 2 innate lymphoid cells in HIV-1 and Mycobacterium leprae co-infected patients. PLoS Negl Trop Dis. 2015;9(9):e0004030.

58. Bernink JH, et al. Interleukin- 12 and -23 control plasticity of $\mathrm{CD} 127(+)$ group 1 and group 3 innate lymphoid cells in the intestinal lamina propria. Immunity. 2015;43(1):146-160.

59. Silver JS, et al. Inflammatory triggers associated with exacerbations of COPD orchestrate plasticity of group 2 innate lymphoid cells in the lungs. Nat Immunol. 2016;17(6):626-635.

60. Biot C, et al. Preexisting BCG-specific T cells improve intravesical immunotherapy for bladder cancer. Sci Transl Med.2012;4(137):137ra72.

61. Powles T, et al. MPDL3280A (anti-PD-L1) treatment leads to clinical activity in metastatic bladder cancer. Nature. 2014;515(7528):558-562.

62. Chevalier MF, Nardelli-Haefliger D, Domingos-Pereira S, Jichlinski P, Derré L. Immunotherapeutic strategies for bladder cancer. Hum Vaccin Immunother. 2014;10(4):977-981.
63. Granot Z, Fridlender ZG. Plasticity beyond cancer cells and the "immunosuppressive switch". Cancer Res. 2015;75(21):4441-4445.

64. Alizadeh D, et al. Doxorubicin eliminates myeloid-derived suppressor cells and enhances the efficacy of adoptive T-cell transfer in breast cancer. Cancer Res. 2014;74(1):104-118.

65. Nefedova Y, Fishman M, Sherman S, Wang X, Beg AA, Gabrilovich DI. Mechanism of alltrans retinoic acid effect on tumor-associated myeloid-derived suppressor cells. Cancer Res. 2007;67(22):11021-11028.

66. Mirza N, et al. All-trans-retinoic acid improves differentiation of myeloid cells and immune response in cancer patients. Cancer Res. 2006;66(18):9299-9307.

67. Spencer SP, et al. Adaptation of innate lymphoid cells to a micronutrient deficiency promotes type 2 barrier immunity. Science. 2014;343(6169):432-437.

68. Tang JE, Wang RJ, Zhong H, Yu B, Chen Y. Vitamin A and risk of bladder cancer: a meta-analysis of epidemiological studies. World J Surg Oncol. 2014;12:130.

69. Hameed DA, el-Metwally TH. The effectiveness of retinoic acid treatment in bladder cancer: impact on recurrence, survival and TGFalpha and VEGF as end-point biomarkers. Cancer Biol Ther. 2008;7(1):92-100.

70. Lamm D, et al. Defining progression in nonmuscle invasive bladder cancer: it is time for a new, standard definition. JUrol. 2014;191(1):20-27.

71. Bubeník J, Baresová M, Viklický V, Jakoubková J, Sainerová H, Donner J. Established cell line of urinary bladder carcinoma (T24) containing tumour-specific antigen. Int J Cancer. 1973;11(3):765-773.

72. Mentzer SJ, Guyre PM, Burakoff SJ, Faller DV. Spontaneous aggregation as a mechanism for human monocyte purification. Cell Immunol. 1986;101(2):312-319.

73. Gupta B, et al. Simultaneous coexpression of memory-related and effector-related genes by individual human CD8 T cells depends on antigen specificity and differentiation. JImmunother. 2012;35(6):488-501.

74. Piehler AP, Grimholt RM, Ovstebø R, Berg JP. Gene expression results in lipopolysaccharidestimulated monocytes depend significantly on the choice of reference genes. BMC Immunol. 2010;11:21.

75. Lawton KA, et al. Analysis of the adult human plasma metabolome. Pharmacogenomics. 2008;9(4):383-397.

76. Pieraerts C, Martin V, Jichlinski P, NardelliHaefliger D, Derre L. Detection of functional antigen-specific $\mathrm{T}$ cells from urine of non-muscle invasive bladder cancer patients. Oncoimmunology. 2012;1(5):694-698.

77. Mathoulin-Pelissier S, Gourgou-Bourgade S, Bonnetain F, Kramar A. Survival end point reporting in randomized cancer clinical trials: a review of major journals. JClin Oncol. 2008;26(22):3721-3726. 